\title{
Matching in the Large: An Experimental Study*
}

\author{
Yan Chen Ming Jiang Onur Kesten Stéphane Robin Min Zhu
}

February 24, 2015

\begin{abstract}
Market size has been predicted to play an influential role in a broad class of economic environments. We study the performance of the Boston and the Deferred Acceptance (DA) mechanism in a laboratory where we increase the market size. Our results show that increasing the market size from 4 to 40 students per match increases participant truth-telling under the DA but decreases it under the Boston mechanism, leading to a decrease in efficiency but no change in the large stability advantage of the DA over the Boston mechanism. Furthermore, increasing the scale to 4,000 students per match has no effect on either individual behavior or mechanism performance. Our results indicate that "large" might be much smaller than predicted.

Keywords: matching, school choice, experiment, scale

JEL Classification: C78, C92, D47, D82

${ }^{*}$ We thank Doug Bernheim, Fuhito Kojima, Erin Krupka, Steve Leider, Sera Linardi, Yusufcan Masatlioglu, Alvin Roth, Marie Claire Villeval, and Alistair Wilson for helpful discussions. Comments from audiences at Carnegie Mellon, Guelph, Lyon, Michigan, Shanghai Jiao Tong, Stanford, Tsinghua, the 2014 International Economic Science Association Meetings (Honolulu, HI), and the 2014 European Economic Science Association Meethings (Prague) are gratefully acknowledged. We thank Desheng Lai, Yunchuan Sun and Yan Shen at Beijing Normal University for letting us use their experimental economics laboratory, and Yefeng Chen for excellent research assistance. The financial support from the National Science Foundation through grant no. SES-0962492 to Chen and Kesten, and from the Agence Nationale de la Recherche under contract ANR-13-BSHS1-0010 to Robin are gratefully acknowledged. This research was approved by the University of Michigan IRB. Chen and Jiang: School of Information, University of Michigan, 105 South State Street, Ann Arbor, MI 48109-2112. Email: yanchen@umich.edu, mjng@umich.edu. Kesten: Tepper School of Business, Carnegie Mellon University, PA 15213. Email: okesten@andrew.cmu.edu. Robin and Zhu: Universitè de Lyon, Lyon, F-69007, France; CNRS, GATE Lyon Saint-Etienne, 93 Chemin des Mouilles, F-69130 Ecully, France. Email: robin@gate.cnrs.fr, zhu@gate.cnrs.fr.
\end{abstract}




\section{Introduction}

Market size has been predicted to play an influential role in a broad class of economic environments. In a large market, existing impossibility results about incentives, welfare, and stability may be overturned, while other existing possibility results may be sharpened to unique solutions. This theoretical phenomenon becomes particularly important for practical market design cases, such as combinatorial auctions, school choice, labor market clearinghouses, course allocation, and kidney exchange, where the market size can range from hundreds to millions. For example, the National Resident Matching Program matches roughly 30,000 doctors and hospitals per year. In another setting, the centralized college admissions systems in Turkey and China match millions of students respectively. Within the United States, school assignments in New York City match nearly 100,000 students per year.

Motivated by practical concerns, there has been a surge of interest in the theoretical study of large matching markets within the last decade by either investigating the asymptotic properties of finite discrete markets or modeling either or both sides of the market as a continuum mass of agents. Within this literature, a significant number of papers have examined the question of whether good incentive and stability properties under the Gale-Shapley deferred acceptance mechanism (Gale and Shapley, 1962) hold as the market size grows, as conjectured in Roth and Peranson (1999). One strand of this literature has shown that, under this mechanism, partner incentives for preference misrepresentations in marriage problems (Immorlica and Mahdian, 2005), college incentives for capacity and preference misrepresentations in college admissions problems (Kojima and Pathak, 2009), and school incentives to disrespect quality improvements in school choice problems (Hatfield et al., 2012) vanish with the market size. Similarly, another line of research has shown that, in a large market satisfying certain regularity assumptions, this mechanism always produces a stable matching in a discrete two-sided matching model, allowing for complementarities (in the form of couples) in the context of the entry-level labor market for U.S. doctors (Kojima et al., 2013; Ashlagi et al., 2011) as well as in continuum models of many-to-one and many-to-many matching (Azevedo and Leshno, 2011; Azevedo and Hatfield, 2013; Che et al., 2013).

In designing practical markets, institutions have relied on economic theory, computation, and controlled laboratory experiments (Roth, 2002). In the school choice reforms in New York City (Abdulkadiroğlu et al., 2005a) and Boston (Abdulkadiroğlu et al., 2005b), for example, matching theorists were directly involved to influence the adoption of the Gale-Shapley deferred acceptance mechanism (Gale and Shapley, 1962; Abdulkadiroğlu and Sönmez, 2003). This represents an improvement over the Boston mechanism, which is severely vulnerable to strategic manipulation in the laboratory (Chen and Sönmez, 2006). Experimental data helped to persuade the Boston public-school authorities to switch from the Boston mechanism to the student-optimal deferred 
acceptance mechanism in 2005 (Abdulkadiroğlu et al., 2005b).

Such institutional redesigns rely on computational as well as laboratory experiments to provide the first data on a theoretically superior mechanism for which field data is not yet available and to compare the performance of different mechanisms at a level of detail that cannot be obtained from field data. Yet another reason for experimentally studying large market dynamics can be attributed to limitations of theoretical analysis. While the theoretical literature characterizes the performance of the canonical matching mechanisms in the large, it is often silent about the question of how large is "large." ${ }^{1}$ To answer this question, empirical, simulation, and experimental work might shed some light.

Matching experiments in particular have been used to study mechanism stability as well as related unraveling issues under different types of clearinghouses (Kagel and Roth, 2000; Niederle and Roth, 2003), performance evaluation of different assignment methods in complete information environments (Chen and Sönmez, 2004; Chen and Kesten, 2014) and the effect of informational and institutional constraints on mechanism performance (Pais and Pintér, 2008; Calsamiglia et al., 2010). Despite the theoretical interest in understanding effects of the market size, attention has not been transferred to the experimental setting for several reasons. First, while laboratory experiments are often compared to a wind tunnel for evaluating new institutions, the scale of a laboratory experiment is often small compared to the corresponding real-world implementations. For example, the largest school choice experiments have 36 subjects per match (Chen and Sönmez, 2006; Calsamiglia et al., 2010), a far cry from the hundreds of thousands of students in Beijing and Shanghai assigned to various high schools each year (He, 2014).

Given the practical importance of understanding how market size impacts mechanism performance, our goal in this paper is to experimentally complement the active and growing literature in matching theory on large market properties within the context of student assignment. To bridge the gap between laboratory experiments and real-world implementations, we compare the performance of two school choice mechanisms in the laboratory when market size increases. Studying large matching markets in the laboratory is of interest for two main reasons. First, large markets often have different theoretical properties than their smaller counterparts. Second, a large market may impact participant behavior due to the complexity of thought it brings to decision making.

We focus our experiment on school choice as it is a widely-debated education policy across the world (Hoxby, 2003; He, 2014), affecting the education experiences and labor market outcomes for millions of students each year. To contribute insight to this debate, we compare and contrast the large market characteristics of the Gale-Shapley deferred acceptance (DA) mechanism to the

\footnotetext{
${ }^{1}$ A notable exception is Rustichini et al. (1994) who show in the context of double auctions six traders of each type are sufficient to obtain efficiency to within one percent. In the context of assignment problems, for a fixed set of object types and a given agent's utility function, Kojima and Manea (2010) calculate the number of copies of each object type needed in order for the probablistic serial mechanism to become strategy-proof.
} 
Boston mechanism. Unlike the DA, which is known in this context to be strategy-proof regardless of market size, the Boston mechanism has been shown to be prone to strategic play in both small and large markets (Kojima and Pathak, 2009; Azevedo and Budish, 2013).

Creating a large market in the laboratory is challenging because of both physical and financial constraints. To address this challenge, we first vary the scale of our all-human sessions from 4 to 40 students per match. To enable random rematching, the latter treatment requires 80 -subject sessions. We then let human subjects play against robots whose strategies are drawn from empirical human strategies from the 40-subject matches, and further increase the scale from 40 to 4,000 students per match. In doing so, we find that when the number of students increases from 4 to 40 , the proportion of truth-telling significantly increases under the DA but decreases under Boston. These results point to nuanced scale effects under each mechanism, though in opposite directions. Our finding of a scale effect under the DA mechanism is unexpected, whereas the increased manipulation under Boston with an increase in scale leads to an even stronger finding than the theoretical view that Boston is likely to remain manipulable in a large market. Further we find that increasing the scale from 40 to 4,000 has no additional effect on the performance of either mechanism. Finally, we find that the matching outcomes under the DA mechanism remain stable as market size increases. Overall, our results indicate that "large" might be much smaller than predicted in the theoretical literature and provide additional support for the replacement of the Boston mechanism with the DA mechanism in practice.

\section{Literature Review}

Incentives for truthful preference revelation in large markets have been studied in matching theory as well as other economic contexts. For example, Roberts and Postlewaite (1976) have shown that the Walrasian mechanism is approximately strategy-proof in a large exchange economy. ${ }^{2}$ In auction theory, Gresik and Satterthwaite (1989), Rustichini et al. (1994), Pesendorfer and Swinkels (2000) and Cripps and Swinkels (2006), and Fudenberg et al. (2007) all show that strategic misreporting vanishes in double auctions in large markets under various informational assumptions. Swinkels (2001) shows a similar result for uniform-price and pay-as-bid auctions.

In indivisible good allocation problems large market arguments have been used to support particular market design approaches. In kidney exchange problems, Roth et al. $(2005,2007)$ show that conducting only small size kidney exchanges is sufficient to achieve full efficiency when the number of incompatible patient-donor pairs is large. When multiple hospitals are involved in an organized exchange program, Ashlagi and Roth (2011) show that it becomes individually ra-

\footnotetext{
${ }^{2}$ Specifically, they show that when equilibrium prices vary continuously with reports, truthtelling is approximately ex-post optimal.
} 
tional for each hospital to participate in the joint exchange program (as opposed to conducting exchanges internally) when the population of hospitals and patients grows large. Finally, in the object assignment context, Kojima and Manea (2010) show that the probabilistic serial mechanism of Bogomolnaia and Moulin (2001) becomes exactly strategy-proof in a sufficiently large finite market.

Relevant to our experimental design, Azevedo and Budish (2013) propose the concept of strategy-proofness in the large (SP-L). They examine the manipulability properties of well known mechanisms as the market size increases and provide a unified view of the large market approaches to incentive issues. In the school choice context, using two different large-market models, Kojima and Pathak (2009) and Azevedo and Budish (2013) both conclude that the Boston mechanism remains manipulable even in a large market. For finite markets Pathak and Sönmez (2013) introduce a metric for ranking different mechanisms according to their manipulability. Interestingly, our experimental results indicate the possibility of varying degrees of manipulability of the same mechanism depending on the scale of the market.

It has been well-documented that the DA mechanism is manipulable in a two-sided matching market (Dubins and Freedman, 1981; Roth, 1982). Some empirical studies and simulations results support the large market predictions of the DA and Boston mechanisms. For example, Roth and Peranson (1999) analyze the NRMP data by conducting simulations on randomly generated simple markets and show that when there is a bound on the length of preferences acceptable to one side of agents, the set of stable matchings becomes small as the market grows. ${ }^{3}$ They observe that, of the more than 20,000 applicants and 3,000-4,000 programs in their study, less than one percent could benefit from truncating preference lists or capacities. During the transition from the previous NRMP algorithm to the new algorithm, less than one percent of applicants and programs are found to be affected by such change, which is comparably small and unsystematic. ${ }^{4}$ By contrast, the DA is strategy-proof in the school choice context where only students are assumed to be strategic agents. Thus the non-manipulability of DA is robust to market size. Using data from the Boston school district prior to the reform, Abdulkadiroğlu et al. (2006) find empirical evidence for both strategically sophisticated and naive play under the Boston mechanism. Based on these theoretical

\footnotetext{
${ }^{3}$ Recently, Azevedo and Leshno (2014) develop a continuum model of the college admissions problem to present a more appealing and general property about the set of stable matchings: that is, a generic continuum economy has a unique stable matching, to which a sequence of the set of stable matchings in large discrete economies converges. Such "core convergence" and uniqueness properties of set of stable matchings have an important implication on other asymptotic properties of the DA mechanism. In particular, Azevedo and Leshno (2014) and Azevedo and Leshno (2011) investigate the DA with single tie-breaking and show that the mechanism is robust to aggregate randomness: whatever tie-breaking results, students will be assigned to almost the same schools under the DA mechanism in a large market.

${ }^{4}$ In the school choice context, Abdulkadiroğlu et al. (2009) also find small changes in matching outcomes when investigating New York City school choice preference data. In several different runs of the DA algorithm, using different lottery outcomes to break the ties in priorities, the aggregate statistics of the match do not vary much.
} 
and empirical results, we expect to see misrepresentation of preferences in the Boston mechanism regardless of market size. We expect no scale effect on the proportion of truth-telling under either mechanism.

With the development of matching theory, a growing number of laboratory experiments have tested mechanism performance as well as participant behavior under different incentives. In one study with 36 students per match, Chen and Sönmez (2006) observe that the proportion of preference manipulation under the Boston mechanism is significantly higher than either the DA or the Top Trading Cycles (TTC) mechanisms. Subsequent studies have examined the impact of different information conditions (Pais and Pintér, 2008), a limit on the number of schools in the rank order list (Calsamiglia et al., 2010), participant risk attitude and preference intensities (Klijn et al., 2013) on participant behavior in school choice experiments. Featherstone and Niederle (2014) observe that the Boston mechanism achieves higher efficiency than the DA mechanism when preferences are private information and when school priorities involve ties which are broken randomly.

Table 1: Representative Experimental Studies of School Choice

\begin{tabular}{llr}
\hline Representative Studies & Mechanisms & \# per match \\
\hline Chen and Sönmez (2006) & Boston, DA, TTC & 36 \\
Pais and Pintér (2008) & Boston, DA, TTC & 5 \\
Calsamiglia et al. (2010) & Boston, DA, TTC & 36 \\
Featherstone and Niederle (2014) & Boston, DA & 5 \\
Klijn et al. (2013) & Boston, DA & 3 \\
Chen and Kesten (2014) & Boston, DA, Chinese Parallel & 4,6 \\
\hline This paper & Boston, DA & $4,40,4,000$ \\
\hline
\end{tabular}

Table 1 summarizes the design features of several representative experimental studies of school choice. In addition to the mechanisms examined in each study, we document the number of students per match. While these experiments use various market sizes, none studies the scale effect. To our knowledge, the only other matching experiment which studies the scale effect is that of Chen and Sönmez (2002), who study house allocations when the number of students increases from 12 to 60 per match. They find that the change in scale has no significant effect on the proportion of truth-telling or participation rate under either TTC or Random Serial Dictatorship with Squatting Rights.

\section{The matching problem and two mechanisms}

A school choice problem (Abdulkadiroğlu and Sönmez, 2003) is comprised of a set of students, each of whom is to be assigned a seat at one school from a set of schools. Each school has a 
number of available seats called the quota for that school. ${ }^{5}$ For each school, there is a strict priority order for all students, and each student has strict preferences over all schools.

Within this context, a matching $\mu$ is a list of assignments such that each student is assigned to one school and the number of students assigned to a particular school does not exceed the quota of that school. A matching $\mu$ is Pareto efficient if there is no other matching which makes all students at least as well off and at least one student better off.

The college admissions problem (Gale and Shapley, 1962) is closely related to the school choice problem. By contrast though, in the college admissions problem, schools have preferences over students, whereas in a school choice problem, schools are objects to be consumed. A key concept in the school choice problem is stability, i.e., there is no unmatched student-school pair $(i, s)$ such that student $i$ prefers school $s$ to his assignment, and either school $s$ has not filled its quota or student $i$ has a higher priority than at least one student $j$ who is enrolled in $s$. In the latter case, we say that student $i$ justifiably envies student $j$ for school $s$. A (school choice) mechanism is a systematic procedure that chooses a matching for each problem. A mechanism is Pareto efficient (stable) if it always selects Pareto efficient (stable) matchings. A mechanism $\varphi$ is strategy-proof if it is a dominant strategy for each student to truthfully report her preferences.

In our study, we focus on two school choice mechanisms. Our first mechanism, the Boston mechanism, is the most common school choice mechanism observed in practice. Its outcome can be calculated via the following algorithm:

Step 1: For each school, consider only those students who have listed it as their first choice. Those students among them with the highest priority for that school are assigned that school up to its quota.

Step $k, k \geq 2$ : Consider the remaining students who are unassigned and the schools that have not filled their quota. For each such school, consider only those students who have listed it as their $k$-th choice. In this group, those students with the highest priority for that school are assigned that school up to its remaining quota.

The algorithm terminates when there are no students left to assign. Importantly, note that the assignments in each step are final. Based on this feature, an important critique of the Boston mechanism is that it gives students strong incentives for gaming through misreported preferences. Because a student who has high priority for a school may lose her priority advantage for that school if she does not list it as her first choice, the Boston mechanism forces students to make hard and potentially costly choices, which leads to a high-stakes game among participants with different

\footnotetext{
${ }^{5} \mathrm{We}$ assume that there are enough seats for all the students, an assumption often met in practice. However, the model is easily modified to accommodate outside options and a shortage of seats.
} 
levels of strategic sophistication (see e.g., Abdulkadiroğlu and Sönmez (2003); Ergin and Sönmez (2006); Chen and Sönmez (2006); Pathak and Sönmez (2008); He (2014)).

In addition to the Boston mechanism, we consider the student-optimal stable mechanism (Gale and Shapley, 1962), which has played a central role in the school choice reforms in Boston and New York City (Abdulkadiroğlu et al., 2005b,a) and, more recently, in Paris. Its outcome can be calculated via the following deferred acceptance (DA) algorithm:

Step 1: Each student applies to her favorite school. Each school tentatively retains those applicants who have the highest priority at that school. The remaining applicants are rejected.

Step $k, k \geq 2$ : Each student rejected from a school at step $k-1$ applies to his next choice school. Each school then tentatively retains those applicants who have the highest priority among the new applicants as well as those tentatively retained at an earlier step. The remaining applicants are rejected.

The algorithm terminates when each student is tentatively retained at some school. Note that, in the DA, assignments in each step are temporary, until the last step. The DA has several desirable theoretical properties, most notably in terms of incentives and stability. First, the DA is strategyproof (Roth, 1982; Dubins and Freedman, 1981). Furthermore, it produces the stable matching that is most favorable to each student. Although its outcome is not necessarily Pareto efficient, it is constrained efficient among the stable mechanisms.

\section{Experimental Design}

We design our experiment to compare the performance of the Boston and the DA mechanisms in a small scale ( $m=4$ per match), a medium scale $(m=40)$, and a large scale matching market ( $m=4,000)$. We adapt our economic environment from the four-school treatment in Chen and Kesten (2014) to capture the key aspects of the school choice problem under complete information.

To study the impact of scale on mechanism performance, our experiment replicates the $m=4$ per match economy to $m=40$ and $m=4000$. We use all-human sessions for the small and medium scale treatments. To make large scale matching market possible in the laboratory, we let human subjects play with computerized agents (robots) programmed to follow empirical strategies previously used by our human subjects under similar conditions. To check for any behavioral differences when human subjects play with other humans versus "empirical" robots, we also design an "empirical" human-robot treatment under the medium scale condition. Lastly, we use a humanvs-truthful-robot design, where all robots always reveal their true preferences, to study subject behavior when there is no uncertainty in opponent strategies and when the human best responses are easy to calculate. 


\subsection{Economic Environment}

In our experiment, there are four schools, $s \in\{A, B, C, D\}$. Each school has 1,10 or 1000 slots, corresponding to the scale of the matching market $m \in\{4,40,4000\}$, respectively. There are four types of students, $i \in\{1,2,3,4\}$, with 1,10 or 1000 of each type, again corresponding to the scale of the matching market.

The payoffs for each type are presented in Table 2. The square brackets, [ ], indicate the district school of the student, where she has higher priority than non-district applicants. Payoffs range from 16 points for the most preferred school to 5 points for the least-preferred school. Each student resides in the district of her second preferred school.

Table 2: Payoff Table

\begin{tabular}{l|cccc}
\hline & A & B & C & D \\
\hline Payoff to Type 1 & {$[11]$} & 7 & 5 & 16 \\
Payoff to Type 2 & 5 & {$[11]$} & 7 & 16 \\
Payoff to Type 3 & 7 & 16 & {$[11]$} & 5 \\
Payoff to Type 4 & 5 & 16 & 7 & {$[11]$} \\
\hline
\end{tabular}

The game preserves the properties of the four-school design in Chen and Kesten (2014): (1) no one lives in her top or bottom choices; (2) the first choice accommodation index, i.e., the proportion of first choices an environment can accommodate, is $1 / 2$, with a fair amount of competition; and (3) the average efficiency under truth-telling is $81 \%$ for DA, reflecting the trade-off between stability and efficiency.

For our all-human small-scale (medium-scale) sessions, there are 12 (80) human subjects of four different types in each session. Subjects are randomly assigned to one of the four types at the beginning of the session and keep their type throughout the experiment. At the beginning of each subsequent round, they are randomly re-matched into three (two) groups of 4 (40) in each smallscale (medium-scale) session. Each 4-participant (40-participant) group has 1 (10) student(s) of each type.

For our human-robot medium-scale (large-scale) sessions, each human subject is paired with 39 (3999) robots. Including the human subject, there are 10 (1000) of each type per match. While a human-robot experimental session can start with any number of human subjects, we control the size of each session to between 19 and 21 human subjects.

In all treatments, we run the experiment for 20 periods to facilitate learning. To investigate whether participant strategies are conditional on their priority, we change the priority queue for each student type every five periods, as indicated in Table 10 in Appendix B. The priority lottery within each type is randomly drawn at the beginning of every five-period block and remains fixed 
for the block. A smaller lottery number indicates a higher priority.

Given this design, we compute the stable outcomes for the school choice game. Using the fact that all stable outcomes are contained between the student-optimal $\left(\mu^{S}\right)$ and school-optimal DA outcome $\left(\mu^{C}\right)$ (Roth and Sotomayor, 1990), $\mu^{S}$ and $\mu^{C}$ coincide in our environment. The unique stable outcome is that each student is assigned to his or her district school. Therefore, while the scale of the market increases, the number of stable outcomes remains as one.

$$
\mu^{S / C}=\mu^{S}=\mu^{C}=\left(\begin{array}{cccc}
1 & 2 & 3 & 4 \\
A & B & C & D
\end{array}\right) ; \mu^{*}=\left(\begin{array}{cccc}
1 & 2 & 3 & 4 \\
A & D & C & B
\end{array}\right) .
$$

Furthermore, in the Boston mechanism, as the Nash Equilibrium outcomes equal to the stable outcomes under complete information (Ergin and Sönmez, 2006), there is a unique Nash equilibrium outcome $\mu^{S / C}$ in our experiment design, in which all students get into their district schools. By contrast, the DA mechanism has one more Nash equilibrium outcome, $\mu^{*}$.

More generally, under our replication method, the number of stable outcomes and the school each type of student gets into do not change with the scale of the market.

Table 3: Truth-telling and Nash Equilibrium Outcomes in the Four-School Game

\begin{tabular}{lllllc}
\hline & \multicolumn{2}{c}{ Truthful Preference Revelation } & & \multicolumn{2}{c}{ Nash Equilibrium Outcomes } \\
\cline { 2 - 3 } \cline { 5 - 5 } & Boston & DA & & Boston & DA \\
\hline Block 1 & not NE & dominant strategy & & \\
Block 2 & not NE & dominant strategy & & $\mu^{S / C}$ & $\left\{\mu^{S / C}, \mu^{*}\right\}$ \\
Block 3 & not NE & dominant strategy & & \\
Block 4 & not NE & dominant strategy & & \\
\hline
\end{tabular}

In addition, we also look at subjects' incentives to reveal their true preferences in large markets. Here, we find that truth-telling is not a Nash equilibrium strategy under the Boston mechanism for any of the priority queues. Table 3 summarizes the properties of this game.

\subsection{Human-vs-Robot Design}

A unique design feature in our study is the pairing of human subjects with computerized agents ("robots") to create a large matching market in the laboratory setting. Previous studies have included robots in their experimental design. In auction experiments, robots follow the dominant strategy in multi-unit Vickrey and English auctions (Kagel and Levin, 2001), Vickrey and iBEA package auctions (Chen and Takeuchi, 2010) and a single-unit Vickrey auction (Davis et al., 2010). 
In Chen and Takeuchi (2010), robots follow a random bidding strategy. Lastly, in a VCM public goods game, robots follow a pre-determined set of actions to eliminate potential other-regarding behavior (Ferraro and Vossler, 2010).

In our experiment, we design two kinds of robots. Our "empirical robots" use strategies previously used by human subjects under similar conditions in the medium scale sessions, i.e., human subjects of the same type, in the same period, with a corresponding priority lottery number. For example, in the medium scale (40 participants per match) sessions, a robot of Type 2 with priority lottery number 15 in period 12, will randomly pick one out of the two choices human subjects of Type 2 with priority lottery number 15 made at period 12. Likewise, in the large-scale (4000 participants per match) sessions, 100 robots with priority lotteries 1401 to 1500 , will randomly pick one of the two choices human subjects of Type 2 with priority lottery number 15 made at period 12, etc. Our human subjects in the human-robot sessions know how their robots counterparts' strategies are drawn and used. To our knowledge, our empirical robot design is new to the experimental literature.

Our second type of robot is our "truthful robot," who always ranks schools truthfully, regardless of its priority, a dominant strategy under the DA mechanism, but a "naïve" one under the Boston mechanism (Ergin and Sönmez, 2006). Again, our human subjects know the robot strategies.

There are three advantages associated with the human-vs-robots design. The first advantage is, of course, the scale. In an all-human experiment, the number of subjects in a group is limited by the capacity of the lab, whereas in a human-vs-robots design, the scale is only limited by the processing power of the computers. The second advantage is to reduce the strategic uncertainty faced by human subjects when they play robots with well defined strategies, which enables the experimenter to study human subjects responses toward opponents of different levels of strategic sophistication. The third advantage is related to statistical independence. Since there is no interaction among human subjects, each human subject is an independent observation.

\subsection{Experimental Procedure}

In each experimental session, each subject is randomly assigned an ID number and seated in front of the corresponding terminal in the laboratory. The experimenter reads the instructions aloud. Subjects are given the opportunities to ask questions, which are answered in public. We check subjects' understanding of the instructions by asking them to answer incentivized review questions at their own pace. After everyone finishes the review questions, the experimenter distributes the answers and goes over the answers in public. Afterwards, subjects go through 20 periods of the school choice experiment. In each period, each subject is asked to submit a full ranking of schools. Robots also submit the rankings of schools under certain strategies in the human-robot sessions. 
After all rankings are submitted, the server allocates the schools and informs each subject of his allocated school and respective payoff. At the end of the 20 periods, each subject fills out a demographics and strategy survey on the computer, and is then paid in private. Each session lasts approximately 90 minutes, of which 30 minutes are devoted to instruction. The experiment is programmed in z-Tree (Fischbacher, 2007) and Python.

Table 4: Features of Experimental Sessions

\begin{tabular}{|c|c|c|c|c|c|}
\hline Mechanisms & Composition & Match size & Robot strategies & \#Sbj. $\times \#$ sessions & Total\# of sbj. \\
\hline \multirow{6}{*}{ Boston } & \multirow{2}{*}{ All-human } & 4 & $\mathrm{n} / \mathrm{a}$ & $12 \times 4$ & 48 \\
\hline & & 40 & $\mathrm{n} / \mathrm{a}$ & $80 \times 2$ & 160 \\
\hline & \multirow{4}{*}{ Human-robot } & \multirow{2}{*}{40} & Empirical & $20 \times 2$ & 40 \\
\hline & & & Truthful & $20 \times 2$ & 40 \\
\hline & & \multirow{2}{*}{4000} & Empirical & $20 \times 2$ & 40 \\
\hline & & & Truthful & $20 \times 2$ & 40 \\
\hline \multirow{6}{*}{ DA } & \multirow{2}{*}{ All-human } & 4 & $\mathrm{n} / \mathrm{a}$ & $12 \times 4$ & 48 \\
\hline & & 40 & $\mathrm{n} / \mathrm{a}$ & $80 \times 2$ & 160 \\
\hline & \multirow{4}{*}{ Human-robot } & \multirow{2}{*}{40} & Empirical & $20 \times 2$ & 40 \\
\hline & & & Truthful & $20 \times 2$ & 40 \\
\hline & & \multirow{2}{*}{4000} & Empirical & $20 \times 2$ & 40 \\
\hline & & & Truthful & $20 \times 2$ & 40 \\
\hline
\end{tabular}

Table 4 summarizes the features of the different experimental sessions. For each mechanism, we conduct four independent sessions for the all-human small-scale treatments, two independent sessions for the all-human intermediate-scale treatments, and two independent sessions for each human-robot intermediate-scale and large-scale treatment, respectively. All sessions are conducted in Chinese at the Experiment Economics Laboratory and the Finance Simulation Laboratory at Beijing Normal University between June 2012 and May 2013. The subjects are students from Beijing Normal University and the Beijing University of Posts and Telecommunications. No subject participates more than once. This gives us a total of 12 independent sessions for the all-human treatments and 320 independent observations for the human-robot treatments. In total, 736 subjects participated in the experiment.

The exchange rate is 5 experiment points for $1 \mathrm{RMB}$ for all sessions. Each subject also receives a participation fee of $5 \mathrm{RMB}$. The average earning (including participation fee) is $63.8 \mathrm{RMB} .{ }^{6}$ The English translations of the experimental instructions can be found in Appendix A.

\footnotetext{
${ }^{6}$ The average wage of part-time work for university students in Beijing was around 30 RMB per hour. The exchange rate at the time of the experiment was around $\$ 1=6 \mathrm{RMB}$.
} 


\section{Results}

In this section, we present results for our experiment, first for the effect of scale on individual behavior and then for the effect of scale on mechanism performance.

\subsection{Individual Behavior}

We first examine the extent to which individuals reveal their preferences truthfully, as well as any patterns in preference manipulation for the two mechanisms when the scale changes. In particular, when there is misrepresentation, we look at a common behavior: listing one's district school as one's first choice.

For the Boston mechanism, we define truth-telling as reporting an entire ranking as identical to one's true preference ranking. For the DA, however, we define truth-telling as reporting a ranking that is identical to the true preference ranking from the first choice to one's district school, as the remaining rankings are irrelevant under DA.

Based on Azevedo and Budish (2013), we expect that participants will misrepresent their preferences under the Boston mechanism regardless of the size of the market, whereas they will reveal their preferences truthfully under the DA mechanism regardless of the size of the market. Therefore, we formulate the following hypotheses on truth-telling:

Hypothesis 1 (Truth-telling: mechanism effect). The proportion of truth-telling under the DA is greater than that under the Boston mechanism, regardless of scale.

Hypothesis 2 (Truth-telling: scale effect). Scale has no effect on the proportion of truth-telling under either the Boston mechanism or the DA mechanism.

We first examine our all-human sessions in treatments with $m=4$, and 40 . Figure 1 presents the proportion of truth-telling (left panel) and district school bias (right panel) for each of the four all-human treatments. The ranking of mechanisms in the truth-telling graph follows the order of DA-40 $>$ DA-4 $>$ BOS-4 $>$ BOS-40, whereas the proportion of district school bias follows the reverse order.

Table 5 presents the results from four probit regressions investigating the scale and mechanism effects in the all-human (upper panel) and human-vs-empirical-robot treatments (lower panel). The dependent variable is truth-telling in specifications (1) and (2), and District School Bias in specifications (3) and (4). In the upper panel, the independent variables include (omitted variables in parentheses): Scale40 (Scale4), DA (Boston), DA $\times$ Scale40, Period, LotteryPosition, LotteryPosition $\times$ DA, LotteryPosition $\times$ Scale40. In each specification, standard errors are clustered at the session level. We summarize the results below. 
Table 5: Truth-telling and district school bias: probit regressions

\begin{tabular}{|c|c|c|c|c|}
\hline \multirow[b]{2}{*}{ All-human treatments } & \multicolumn{2}{|c|}{ Truth-telling } & \multicolumn{2}{|c|}{ District School Bias } \\
\hline & (1) & (2) & (3) & (4) \\
\hline Scale 40 & $\begin{array}{c}-0.081 * * * \\
(0.024)\end{array}$ & $\begin{array}{c}-0.122 * * * \\
(0.033)\end{array}$ & $\begin{array}{c}0.110 * * * \\
(0.033)\end{array}$ & $\begin{array}{c}0.166 * * * \\
(0.041)\end{array}$ \\
\hline DA & $\begin{array}{c}0.132 * * * \\
(0.031)\end{array}$ & $\begin{array}{c}0.111 * * * \\
(0.025)\end{array}$ & $\begin{array}{c}-0.211 * * * \\
(0.046)\end{array}$ & $\begin{array}{c}-0.205^{* * *} * \\
(0.046)\end{array}$ \\
\hline $\mathrm{DA} \times$ Scale 40 & $\begin{array}{c}0.146 * * * \\
(0.041)\end{array}$ & $\begin{array}{c}0.143 * * * \\
(0.041)\end{array}$ & $\begin{array}{c}-0.098 * * \\
(0.049)\end{array}$ & $\begin{array}{c}-0.094^{*} \\
(0.049)\end{array}$ \\
\hline Period & & $\begin{array}{c}0.004 * * * \\
(0.001)\end{array}$ & & $\begin{array}{c}-0.004 * * \\
(0.002)\end{array}$ \\
\hline LotteryPosition & & $\begin{array}{c}-0.125 * * * \\
(0.010)\end{array}$ & & $\begin{array}{c}0.127 * * * \\
(0.012)\end{array}$ \\
\hline LotteryPosition $\times$ DA & & $\begin{array}{c}0.009 \\
(0.006)\end{array}$ & & $\begin{array}{l}-0.003 \\
(0.009)\end{array}$ \\
\hline LotteryPosition $\times$ Scale 40 & & $\begin{array}{c}0.017 \\
(0.012)\end{array}$ & & $\begin{array}{l}-0.022 * \\
(0.013)\end{array}$ \\
\hline No. of observations & 8,320 & 8,320 & 8,320 & 8,320 \\
\hline Human-vs-e-robots treatments & $(1)$ & $(2)$ & $(3)$ & $(4)$ \\
\hline Scale4K & $\begin{array}{c}-0.018 \\
(0.069)\end{array}$ & $\begin{array}{c}0.020 \\
(0.083)\end{array}$ & $\begin{array}{c}0.012 \\
(0.066)\end{array}$ & $\begin{array}{c}-0.011 \\
(0.080)\end{array}$ \\
\hline DA & $\begin{array}{c}0.160 * * \\
(0.064)\end{array}$ & $\begin{array}{c}0.040 \\
(0.082)\end{array}$ & $\begin{array}{c}-0.229 * * * \\
(0.061)\end{array}$ & $\begin{array}{l}-0.128 \\
(0.078)\end{array}$ \\
\hline $\mathrm{DA} \times$ Scale $4 \mathrm{~K}$ & $\begin{array}{c}0.063 \\
(0.091)\end{array}$ & $\begin{array}{c}0.064 \\
(0.091)\end{array}$ & $\begin{array}{c}-0.072 \\
(0.088)\end{array}$ & $\begin{array}{l}-0.073 \\
(0.088)\end{array}$ \\
\hline Period & & $\begin{array}{c}0.003^{*} \\
(0.002)\end{array}$ & & $\begin{array}{c}-0.003 * \\
(0.002)\end{array}$ \\
\hline LotteryPosition & & $\begin{array}{c}-0.097 * * * \\
(0.017)\end{array}$ & & $\begin{array}{c}0.090 * * * \\
(0.015)\end{array}$ \\
\hline LotteryPosition $\times$ DA & & $\begin{array}{c}0.047 * * \\
(0.020)\end{array}$ & & $\begin{array}{c}-0.039 * * \\
(0.019)\end{array}$ \\
\hline LotteryPosition $\times$ Scale $4 \mathrm{~K}$ & & $\begin{array}{c}-0.016 \\
(0.020) \\
\end{array}$ & & $\begin{array}{c}0.009 \\
(0.019) \\
\end{array}$ \\
\hline No. of Human Observations & 3,200 & 3,200 & 3,200 & 3,200 \\
\hline
\end{tabular}

Notes: Standard errors in parentheses are clustered at the session (individual) level for the allhuman (human-vs-empirical-robot) treatments; coefficients are marginal effects. $* * * \mathrm{p}<0.01$, ** $\mathrm{p}<0.05, * \mathrm{p}<0.1$. 


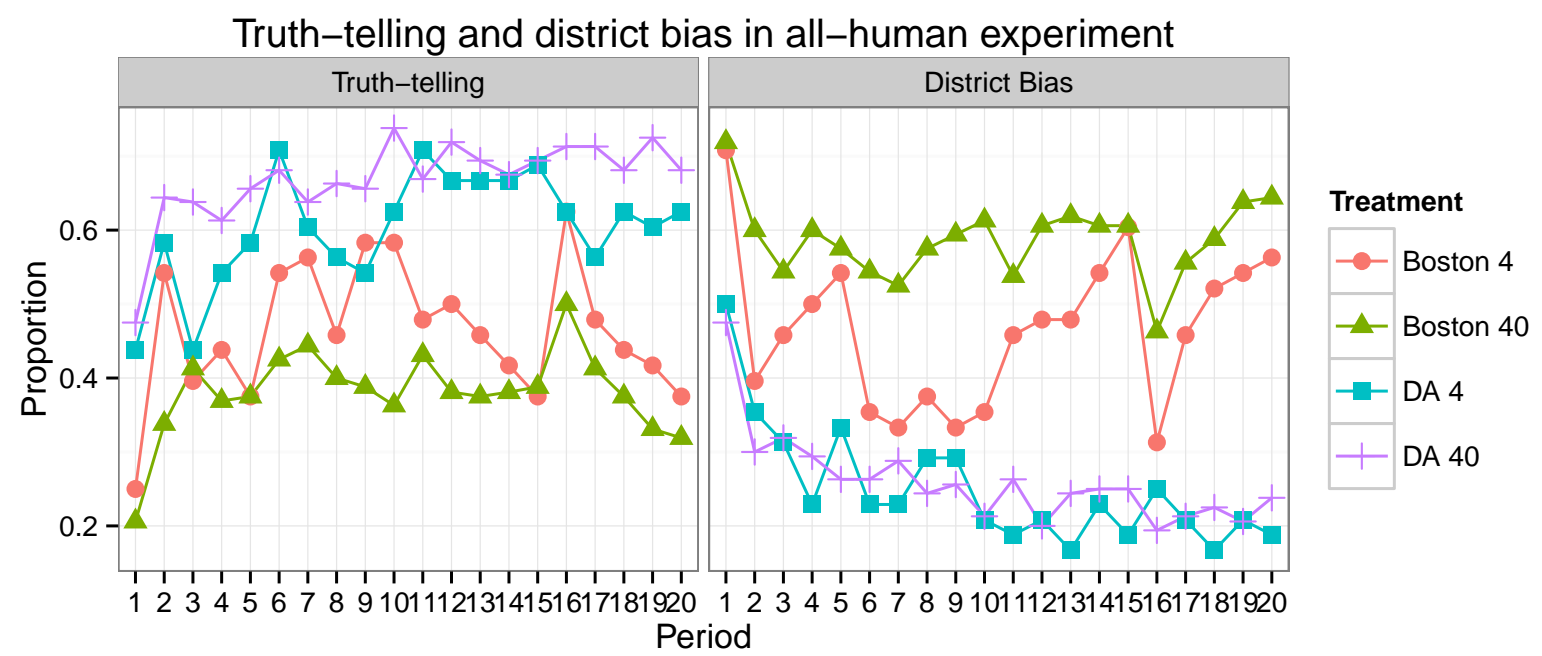

Figure 1: Truth-telling and district school bias in all-human treatments

Result 1 (Truth-telling: mechanism effect, all human). The proportion of truth-telling is 13\% (28\%) higher under the DA mechanism than under the Boston mechanism in the 4(40)-student environment.

Support. In specification (1) of the upper panel of Table 5, we see that the coefficients of DA as well as DA $\times$ Scale40 are positive and significant, indicating that truth-telling under DA in the 4student (40-student) matches is $13 \%$ (28\%) higher compared to that in the corresponding sessions under the Boston mechanism.

By Result 1, we reject the null in favor of Hypothesis 1 under both $m=4$ and $m=40$. Our mechanism effect under $m=4$ is consistent with the identical treatments in Chen and Kesten (2014), as well as with prior experimental studies of school choice in comparable markets. We also note that the gap between the two mechanisms more than doubles when we increase the scale by a factor of 10 . This leads to our next result.

Result 2 (Truth-telling: scale effect $4 \rightarrow 40$ ). The proportion of truth-telling under the DA (Boston) mechanism significantly increases (decreases) when the number of students per match increases from 4 to 40.

Support. In specification (1) of the upper panel of Table 5, the coefficient of DA $\times$ Scale40 is positive and significant, indicating that truth-telling in the 40-student matches under DA increases by $7 \%$ compared to that in the 4 -student matches. In comparison, the coefficient of Scale 40 is negative and significant, indicating that truth-telling in the 40 -student sessions under Boston decreases by $8 \%$ compared to that in the 4 -student sessions. 
By Result 2, we reject Hypothesis 2 that scale has no effect on truth-telling under either mechanism. Indeed, we find a significant and sizeable scale effect for both mechanisms. Specifically, when the number of students per match increases from 4 to 40, participants exhibit less manipulation of the DA but more manipulation of the Boston mechanism. Consequently, we conclude that scale magnifies the performance gap between the two mechanisms.

We next explore the patterns of manipulation behind our scale effect. As documented in several experiments (Chen and Sönmez, 2006; Calsamiglia et al., 2010), the most prevalent form of manipulation in school choice experiments is the district school bias, where a student gives her district school a higher ranking than its true ranking. In particular, when a district school is ranked as the top choice, a participant is guaranteed to be assigned to this school under the Boston mechanism. In our experiment, we find significant scale and mechanism effects in this type of manipulation.

Result 3 (District school bias: mechanism and scale effects). The proportion of DSB under the Boston mechanism is $21 \%$ (30\%) higher than that under the DA mechanism in the 4-student (40student) treatment. Under the DA (Boston) mechanism, the proportion of district school bias increases by $1 \%$ (11\%) when the size of the match increases from 4 to 40.

Support. In specification (3) in the upper panel of Table 5, the coefficient of DA is $-0.21(p<$ $0.01)$, that of DA $\times$ Scale40 is $-0.098(p<0.05)$, and that of Scale40 is $0.11(p<0.01)$.

Furthermore, we observe a significant, albeit moderate, learning effect across periods, i.e., the proportion of truth-telling (DSB) increases (decreases) by about $0.4 \%$ per period $(p<0.01$, specifications (2) and (4)). We also find that, under the Boston mechanism, a one point increase in the lottery position decreases the likelihood of truth-telling by $12.5 \%$ ( $p<0.01$, specification (2)), but increases the likelihood of DSB by $12.7 \%$ ( $p<0.01$, specification (4)), indicating a tendency to seek secure allocations as one's priority deteriorates. In comparison, such effect under the DA mechanism is smaller ( $p<0.01$, specifications (2) and (4)).

Our finding of a significant scale effect $(4 \rightarrow 40)$ under both mechanisms is consistent with individual players adopting secure strategies as the size of the market increases. Under the Boston mechanism, district school bias guarantees that a player is matched with her second choice, regardless of what others do. Similarly, truth-telling is a weakly dominant strategy under the DA mechanism. Thus, regardless of what others do, a player cannot be worse off adopting this strategy. However, the unstable Nash equilibrium outcome under the DA requires half of the players to coordinate their manipulation. Doing so becomes increasingly difficult when the scale increases. Therefore, when the market size increases by a factor of 10 , we observe increased adoption of the dominant strategy under the DA mechanism and increased district school bias under the Boston mechanism. 
Next, we investigate the scale effect when the number of students per match increases from 40 to 4,000. As we transition from our all-human to human-vs-empirical-robot sessions, we note that our human subjects have the same behavioral responses in the all-human $m=40$ treatments as in the human-vs-39-empirical-robots treatments. Table 12 in Appendix B presents $p$-values computed from Kolmogorov-Smirnov tests, comparing the probability distributions of submitted preference rankings between the all-human 40-student and human-versus-39-empirical-robots sessions for each mechanism period by period. These results show no statistically significant difference in participant behaviors in the respective treatments. These results indicate that humans do not respond differently when playing against robots whose strategies are drawn from the same human population.

In our human-vs-empirical-robot treatments, each human participant plays against either 39 or 3,999 robots whose strategies are randomly drawn from the all-human 40-student treatments. Figure 2 presents the proportion of truth-telling (left panel) and district school bias (right panel) for each of the human-vs-empirical-robot treatments. The ranking of treatments in the truth-telling graph follows the order of DA-4000 $>$ DA-40 $>$ BOS-40 $\sim$ BOS-4000, whereas the proportion of district school bias follows the reversed order.

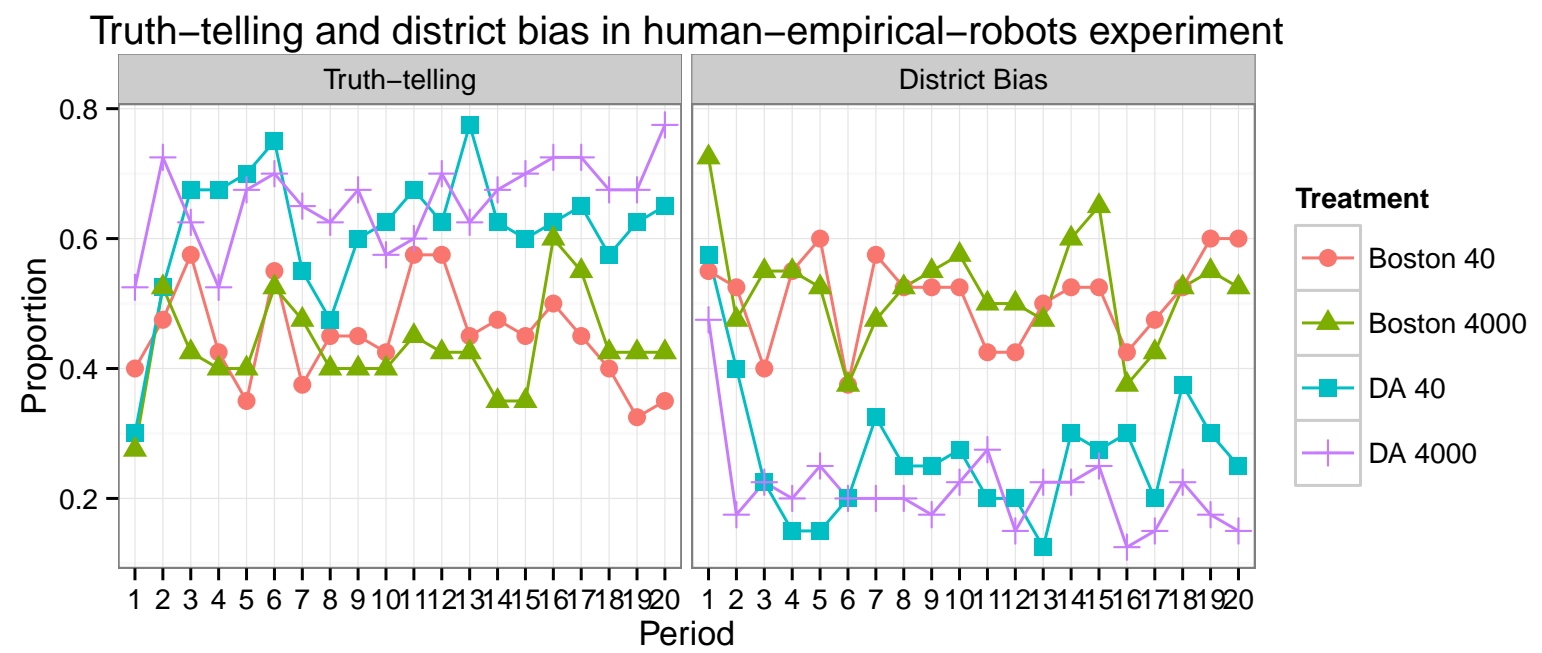

Figure 2: Truth-telling and district school bias in human-vs-empirical-robot treatments

The lower panel of Table 5 presents the results of four probit regressions investigating the scale and mechanism effects in the human-vs-empirical-robot treatments. The dependent variable is Truth-telling in specifications (1) and (2), and District School Bias in (3) and (4). The independent variables include (omitted variables in parentheses): Scale4K (Scale40), DA (Boston), DA $\times$ Scale4K, Period, LotteryPosition, LotteryPosition $\times$ DA, LotteryPosition $\times$ Scale4K. In 
each specification, standard errors are clustered at the individual subject level as each subject is an independent observation. We summarize the results below.

Result 4 (Truth-telling: mechanism effects, human-vs-empirical-robots). The proportion of truthtelling is significantly higher under the DA than under the Boston mechanism in the human-vsempirical-robots treatments. Furthermore, this mechanism effect works through the subjects' lottery positions, i.e., a one-position increase in lottery position decreases the likelihood of truthtelling by $10 \%$ (5\%) under the Boston (DA) mechanisms.

Support. In specification (1) of Table 5 (lower panel), the coefficient of DA is positive and significant, indicating that the proportion of truth-telling under the DA in the 40-student sessions is about $16 \%$ higher compared to that in the corresponding sessions under the Boston mechanism. The effect of the lottery position under the Boston mechanism, indicated by the coefficient of LotteryPosition, is $-0.097(p<0.01)$, while the effect under the DA mechanism is indicated by the sum of the coefficients of LotteryPosition and LotteryPosition $\times$ DA $(0.047, p<0.05)$ in specification (2).

By Result 4, we again reject the null in favor of Hypothesis 1 in our human-vs-empirical-robots treatments. However, we note that the coefficient of Scale4K $(\mathrm{DA} \times \mathrm{Scale} 4 \mathrm{~K})$ is negative (positive) but insignificant, indicating a lack of scale effect. This leads to our next result.

Result 5 (Truth-telling: scale effect $40 \rightarrow 4 K$ ). The proportion of truth-telling under the DA (Boston) mechanism increases (decreases) when the size of the match increases from 40 to 4,000; however, this effect is statistically insignificant.

Support. In specification (1) of Table 5 (lower panel), the coefficient of Scale4K $(\mathrm{DA} \times \mathrm{Scale} 4 \mathrm{~K})$ is negative (positive) but insignificant, indicating that the proportion of truth-telling in the 40student sessions under Boston (DA) is not different from that in the 4000-student sessions.

By Result 5, we fail to reject Hypothesis 2 when the scale increases from 40 to 4,000 students per match in our human-vs-empirical-robots treatments.

Finally, looking at specification (3) in the lower panel of Table 5, we see that the proportion of district school bias under the DA mechanism is $23 \%$ less than that under the Boston mechanism when the size is $40(p<0.01)$, comparable to the magnitude of bias in the all-human 40-student per match treatments in Result 3. Additionally, we have analyzed human subjects' likelihood of best response in each treatment, and find both a mechanism (more best responses under the DA mechanism) and a scale effect (more best responses with increased scale). This analysis is relegated to Appendix $\mathrm{C}$ due to space constraints. 


\subsection{Aggregate Performance}

In this section, we examine the scale and mechanism effects on measures of aggregate performance: the proportion of students admitted by both their reported and true first choice schools, individual rationality, efficiency and stability. For each measure, we first compare the all-human treatments ( $m=4,40)$, and then compare the human-vs-empirical-robots treatments $(m=40,4000)$.

\subsubsection{First Choice Accommodation and Individual Rationality}

We first look at the first-choice accommodation rate, differentiating between the proportion of students admitted by their true versus reported first choice schools.

Based on Ergin and Sönmez (2006), we expect that a higher proportion of participants will receive their true first choices under the DA mechanism compared to the Boston mechanism. In contrast, we expect that a larger proportion will receive their reported top choices under the Boston mechanism (Chen and Kesten, 2014).

Hypothesis 3 (First-choice accommodation: mechanism effect). If subjects play Nash equilibrium strategies, a higher proportion will receive (a) their true first choices under the DA mechanism, and (b) their reported first choices under the Boston mechanism.

Figures 4 and 5 in Appendix B present the first-choice accommodation rate for the all-human and the human-vs-empirical-robots treatments, respectively, comparing the proportion of subjects receiving their reported (top panel) and true first choices (bottom panel). We see that a greater proportion receive their reported top choice under the Boston mechanism, but the gap between the two mechanisms is much smaller when examining subjects' true top choices.

Table 6 reports the results from four probit specifications for students' true (1-2) and reported first choices (3-4) for both the all-human (upper panel) and the human-vs-empirical-robots treatments (lower panel). The independent variables for the upper panel include: Scale40 (Scale4), DA (Boston), DA $\times$ Scale40, Period, LotteryPosition, LotteryPosition $\times$ DA, and LotteryPosition $\times$ Scale40. The lower panel reports similar specifications except for Scale4K (Scale40). In the all-human sessions, we cluster the standard errors at the session level, whereas in the human-vsempirical-robots treatment, we cluster the standard errors at the individual level as each human subject interacts with only robots.

Result 6 (First-choice accommodation: mechanism effect). Regardless of scale, both the reported and the true first-choice accommodation rates are significantly higher under the Boston than the DA mechanism.

Support. In the upper panel of Table 6, the coefficients of DA are negative and significant $(p<$ 0.01 in each of the four specifications), indicating a 5.8\% (26.6\%) reduction in receiving one's true 
Table 6: First choice accommodation: Probit regressions

\begin{tabular}{|c|c|c|c|c|}
\hline Dependent Variable: & \multicolumn{2}{|c|}{ True First Choice } & \multicolumn{2}{|c|}{ Reported First Choice } \\
\hline All-Human Treatments & (1) & (2) & (3) & (4) \\
\hline Scale 40 & $\begin{array}{c}-0.042 * * * \\
(0.010)\end{array}$ & $\begin{array}{c}-0.035^{* *} \\
(0.014)\end{array}$ & $\begin{array}{c}0.055 * * \\
(0.028)\end{array}$ & $\begin{array}{c}0.030 \\
(0.036)\end{array}$ \\
\hline DA & $\begin{array}{c}-0.058 * * * \\
(0.016)\end{array}$ & $\begin{array}{c}-0.067 * * * \\
(0.019)\end{array}$ & $\begin{array}{c}-0.266 * * * \\
(0.033)\end{array}$ & $\begin{array}{c}-0.279 * * * \\
(0.039)\end{array}$ \\
\hline $\mathrm{DA} \times$ Scale 40 & $\begin{array}{c}-0.044 * * \\
(0.020)\end{array}$ & $\begin{array}{c}-0.044 * * \\
(0.018)\end{array}$ & $\begin{array}{c}-0.104 * * * \\
(0.039)\end{array}$ & $\begin{array}{c}-0.107 * * * \\
(0.039)\end{array}$ \\
\hline Period & & $\begin{array}{c}0.000 \\
(0.001)\end{array}$ & & $\begin{array}{c}-0.005 * * * \\
(0.001)\end{array}$ \\
\hline LotteryPosition & & $\begin{array}{c}-0.034 * * * \\
(0.004)\end{array}$ & & $\begin{array}{c}0.066^{* * * *} \\
(0.013)\end{array}$ \\
\hline Lottery $\times$ DA & & $\begin{array}{c}0.005 \\
(0.007)\end{array}$ & & $\begin{array}{c}0.006 \\
(0.011)\end{array}$ \\
\hline Lottery $\times$ Scale 40 & & $\begin{array}{c}-0.003 \\
(0.005)\end{array}$ & & $\begin{array}{c}0.010 \\
(0.015)\end{array}$ \\
\hline Number of observations & 8,320 & 8,320 & 8,320 & 8,320 \\
\hline Human-vs-e-Robots & $(1)$ & $(2)$ & (3) & (4) \\
\hline Scale4K & $\begin{array}{c}0.007 \\
(0.019)\end{array}$ & $\begin{array}{c}0.010 \\
(0.029)\end{array}$ & $\begin{array}{c}0.025 \\
(0.052)\end{array}$ & $\begin{array}{c}0.010 \\
(0.066)\end{array}$ \\
\hline DA & $\begin{array}{c}-0.087 * * * \\
(0.021)\end{array}$ & $\begin{array}{l}-0.057 * \\
(0.032)\end{array}$ & $\begin{array}{c}-0.279 * * * \\
(0.050)\end{array}$ & $\begin{array}{c}-0.214 * * * \\
(0.067)\end{array}$ \\
\hline $\mathrm{DA} \times$ Scale $4 \mathrm{~K}$ & $\begin{array}{l}-0.025 \\
(0.030)\end{array}$ & $\begin{array}{l}-0.023 \\
(0.030)\end{array}$ & $\begin{array}{l}-0.089 \\
(0.073)\end{array}$ & $\begin{array}{l}-0.090 \\
(0.073)\end{array}$ \\
\hline Period & & $\begin{array}{l}-0.001 \\
(0.001)\end{array}$ & & $\begin{array}{c}-0.005 * * * \\
(0.002)\end{array}$ \\
\hline LotteryPosition & & $\begin{array}{c}-0.031 * * * \\
(0.008)\end{array}$ & & $\begin{array}{c}0.054 * * * \\
(0.016)\end{array}$ \\
\hline Lottery $\times$ DA & & $\begin{array}{l}-0.017 \\
(0.012)\end{array}$ & & $\begin{array}{l}-0.026 \\
(0.020)\end{array}$ \\
\hline Lottery $\times$ Scale $4 \mathrm{~K}$ & & $\begin{array}{l}-0.002 \\
(0.010)\end{array}$ & & $\begin{array}{c}0.006 \\
(0.020)\end{array}$ \\
\hline Number of human obs. & 3,200 & 3,200 & 3,200 & 3,200 \\
\hline
\end{tabular}

Notes: Standard errors in parentheses are clustered at the session (individual) level for the allhuman (human-vs-empirical-robots) treatments; reporting marginal effects. $* * * \mathrm{p}<0.01$, ** $\mathrm{p}<$ $0.05, * \mathrm{p}<0.1$ 
(reported) first choice under the DA in the $m=4$ treatments in specification 1 (3). Furthermore, the coefficients of DA $\times$ Scale40 are negative and significant $(p<0.05$ in specifications (1) and (2), $p<0.01$ in specifications (3) and (4)), indicating a further reduction of $4.4 \%$ (10.4\%) of students receiving their true (reported) first choice under the DA in the $m=40$ treatments.

In comparison, from the lower panel of Table 6, we see that the coefficients of DA are also negative and significant ( $p<0.01$ in each of the six specifications), indicating a $8.7 \%(27.9 \%)$ reduction in receiving one's true (reported) first choice under the DA in the $m=40$ treatments in specification 1 (3). However, the coefficients of DA $\times$ Scale $4 \mathrm{~K}$ are negative but insignificant $(p>$ 0.10 ), indicating no further reduction in receiving one's first choice under $m=4,000$ treatments.

By Result 6, we reject the null in favor of Hypothesis 3 (b), but fail to reject the null in favor of Hypothesis 3 (a). Our findings confirm the theoretical prediction that the Boston mechanism places heavy weight on how students report their first choices. In our study, the true first-choice accommodation rate is lower under the DA because the dominant strategy equilibrium assigns everyone to his second choice.

Result 7 (First-choice accommodation: scale effect). The increase in scale from $4 \rightarrow 40$ significantly decreases (increases) the true (reported) first-choice accommodation rate by $4 \%$ (5\%) under the Boston mechanism, but significantly decreases the rate by $8 \%(5 \%)$ under the DA mechanism. By contrast, the increase in scale from $40 \rightarrow$ 4, 000 has no significant effect on either first-choice accommodation rate.

Support. In the upper panel of Table 6, the coefficients of Scale40 are -0.04 ( $p<0.01$ in specification (1), $p<0.05$ in (2)), and $0.055(p<0.05)$ in specification (3). Furthermore, the coefficients of DA $\times$ Scale40 are $-0.044(p<0.05)$ in specifications $(1)$ and $(2)$, and $-0.104(p<0.01)$ in specification (3) and (4). In comparison, in the lower panel of Table 6, none of the coefficients of Scale $4 \mathrm{~K}$ or DA $\times$ Scale $4 \mathrm{~K}$ is significant $(p>0.10)$.

Result 7 is consistent with the scale effect on truth-telling (Results 2 and 5). Increased district school bias under the Boston mechanism under the medium scale leads to a significantly higher (lower) proportion receiving their reported (true) first choice schools. By contrast, increased truthtelling under the DA leads to an increased proportion receiving their second choice, which is the dominant strategy equilibrium.

We next examine any scale and mechanism effect on individual rationality, i.e., the proportion of students placed at a school which is at least as good as her district school. Since students are guaranteed a seat at their district schools under the DA mechanism by playing the truth-telling strategy, which is not the case under the Boston mechanism, we have the following hypothesis. 
Hypothesis 4 (Individual rationality). More students will receive individually rational allocations under the DA than under the Boston mechanism regardless of scale.

Table 7: Individual rationality in all-human and empirical robots treatments: Probit regressions

\begin{tabular}{rcccc}
\hline Dependent Variable: & \multicolumn{4}{c}{ Individual Rationality } \\
\hline Treatments: & \multicolumn{2}{c}{ All-human } & \multicolumn{1}{c}{ Human-vs-empirical-robots } \\
\hline & $(1)$ & $(2)$ & $(3)$ & $(4)$ \\
\hline Scale & $0.027^{* * *}$ & $0.048^{* * *}$ & -0.015 & -0.008 \\
& $(0.009)$ & $(0.018)$ & $(0.018)$ & $(0.027)$ \\
DA & $0.072^{* * *}$ & $0.105^{* * *}$ & $0.048^{* * *}$ & $0.094^{* * *}$ \\
& $(0.016)$ & $(0.026)$ & $(0.018)$ & $(0.028)$ \\
DA $\times$ Scale & -0.007 & -0.007 & 0.004 & 0.005 \\
& $(0.023)$ & $(0.022)$ & $(0.025)$ & $(0.025)$ \\
Period & & $0.001^{* *}$ & & $0.003^{* * *}$ \\
& & $(0.000)$ & & $(0.001)$ \\
Lottery & & $0.029^{* * *}$ & & $0.025^{* * *}$ \\
& & $(0.005)$ & & $-0.007)$ \\
Lottery $\times$ DA & & $-0.015^{*}$ & & $-0.020^{* *}$ \\
& & $(0.008)$ & & $-0.008)$ \\
Lottery $\times$ Scale & \multicolumn{5}{c}{-0.009} & & $(0.003)$ \\
\hline Observations & 8,320 & $(0.006)$ & & 3,200 \\
\hline
\end{tabular}

Notes: Standard errors in parentheses are clustered at the session (individual) level for the allhuman (human-vs-empirical-robots) treatments; reporting marginal effects. $* * * \mathrm{p}<0.01$, ** $\mathrm{p}<$ $0.05, * \mathrm{p}<0.1$

Table 7 presents four probit specifications for the all-human (1-2) and human-vs-empiricalrobots treatments (3-4), whereas Figure 6 in Appendix B presents the proportion of individual rational allocations in each treatment.

Result 8 (Individual rationality: mechanism and scale effects). Regardless of scale, the proportion of individually rational allocations is significantly higher under the DA than the Boston mechanism. The increase in scale from $4 \rightarrow 40$ significantly increases the proportion of individually rational allocations under both mechanisms.

Support. In Table 7, the coefficients of DA are 0.072 and 0.048 for all-human and human-vsempirical robots treatments respectively ( $p<0.01$ in all specifications). In addition, increasing the scale in all-human treatment from 4 to 40 also increase the proportion of students receiving individually rational allocations for both mechanisms $(p<0.01)$. 


\subsubsection{Efficiency and Stability}

To study the effect of scale on mechanism efficiency, we define payoff-based efficiency in our allhuman treatments as the sum of the individual payoffs in each match in each period, normalized according to the following formula:

$$
\text { Payoff-based Efficiency }=\frac{\text { Actual sum of payoffs }- \text { Minimum sum of payoffs }}{\text { Maximum sum of payoffs }- \text { Minimum sum of payoffs }} \text {. }
$$

In our environment, the minimum sum of payoffs is 240 for the 40 -student environment, and 24,000 for the 4,000-student environment. Likewise, the maximum sum of payoffs is 540 for the 40-student environment, and 54,000 for the 4,000-student environment.

In the human-vs-empirical-robots treatments, the equivalence of the payoff-based efficiency is the human players' expected payoffs. Specifically, for each human subject in each period, we recombine the subject with 39 (or 3999) group members in the same period, of the same type, and with the same (or corresponding) lottery number, taken from the all-human session. While there are $2^{39}\left(2^{3999}\right)$ possible recombinations, to reduce computation, we randomly generate 2000 groups for each subject. We then estimate the expected payoff for each human subject by averaging her payoffs over the 2000 group recombinations.

Table 8: Payoff-based efficiency and justified envy in all-human treatments: OLS regressions

\begin{tabular}{rcccc}
\hline Dependent Variable: & \multicolumn{2}{c}{ Payoff-based Efficiency } & \multicolumn{2}{c}{ Justified Envy } \\
\hline & $(1)$ & $(2)$ & $(3)$ & $(4)$ \\
\hline Scale40 & $-0.014^{* * *}$ & $-0.014^{* * *}$ & -0.006 & -0.006 \\
& $(0.004)$ & $(0.004)$ & $(0.022)$ & $(0.022)$ \\
DA & 0.004 & 0.004 & $-0.076^{* * *}$ & $-0.076^{* * *}$ \\
& $(0.011)$ & $(0.011)$ & $(0.021)$ & $(0.021)$ \\
DA $\times$ Scale40 & -0.019 & -0.019 & -0.024 & -0.024 \\
& $(0.012)$ & $(0.012)$ & $(0.028)$ & $(0.028)$ \\
Period & & 0.000 & & 0.000 \\
& & $(0.001)$ & & $(0.001)$ \\
Constant & $0.690^{* * *}$ & $0.688^{* * *}$ & $0.140^{* * *}$ & $0.141^{* * *}$ \\
& $(0.003)$ & $(0.012)$ & $(0.018)$ & $(0.024)$ \\
\hline Observations & 640 & 640 & 640 & 640 \\
R-squared & 0.018 & 0.019 & 0.096 & 0.096 \\
\hline
\end{tabular}

Notes: Standard errors in parentheses are clustered at the session level. $* * * p<0.01, * * p<0.05$, $* \mathrm{p}<0.1$ 
Tables 8 and 9 report the results of our OLS regression analysis of efficiency and justified envy in the all-human and human-vs-empirical-robots treatments, respectively. Specifications (1) and (2) in Table 8 indicate that increasing the scale from 4 to 40 significantly reduces payoff-based efficiency under the Boston mechanism $(-0.014, p<0.01)$, but has no further significant effect on the DA. Similarly, specifications (1) and (2) in Table 9 indicate that further increasing the scale from 40 to 4000 has no effect on the efficiency of either mechanism.

Result 9 (Efficiency: scale effect). From $4 \rightarrow 40$, payoff-based efficiency under the Boston (DA) mechanism decreases by $1.4 \%$ (3.3\%). Further increasing the scale to 4000 has no additional effect on the efficiency of either mechanism.

Support. In specifications (1) and (2) of Table 8, the coefficients for both Scale40 (-0.014, $p<$ $0.01)$ and Scale $40+\mathrm{DA} \times \mathrm{Scale} 40(-0.033, p=0.0147)$ are negative and significant. In comparison, none of the coefficients for the scale variables is significant in specifications (1) and (2) in Table 9.

The small but significant decrease in efficiency is likely due to the increased manipulation of preferences under the Boston mechanism, as the scale increases from 4 to 40 . While the efficiency comparisons between the DA and the Boston mechanism depend on the environment, in our environment, we do not observe a mechanism effect.

Finally, we investigate the mechanism and scale effect on stability. Empirically, we evaluate mechanism stability by calculating the proportion of students in each group who exhibit justified envy toward at least one other student. As the DA mechanism is stable while the Boston mechanism is not, we expect that the proportion of students exhibiting justified envy will be lower under the DA at any given scale.

Hypothesis 5 (Stability: mechanism effect). The proportion of students exhibiting justified envy is lower under the DA than under the Boston mechanism at any given scale.

Theoretical work on the scale effect implies that, in general, the set of stable matchings decreases for both mechanisms when the scale increases. However, since we have a unique stable outcome in our environment, we do not expect any scale effect on stability.

Hypothesis 6 (Stability: scale effect). Scale has no effect on the proportion of students exhibiting justified envy under either the Boston or the DA mechanism.

Figure 3 presents the results for the average proportion of students with justified envy in our allhuman treatments (left panel) and the average expected probability of having justified envy in the human-vs-empirical-robots (right panel) treatments. For the human-vs-empirical-robot treatments, we investigate stability through the use of simulations. More specifically, we randomly generate 
Table 9: Efficiency and justified envy in human-vs-empirical-robots treatments: OLS regressions

\begin{tabular}{rllll}
\hline Dependent Variable: & \multicolumn{2}{c}{ Expected Payoff } & \multicolumn{2}{c}{ Justified Envy } \\
\hline & $(1)$ & $(2)$ & $(3)$ & $(4)$ \\
\hline Scale4K & -0.052 & 0.004 & 0.012 & -0.001 \\
& $(0.224)$ & $(0.346)$ & $(0.023)$ & $(0.039)$ \\
DA & $-0.277^{*}$ & -0.231 & $-0.063^{* * *}$ & $-0.135^{* * *}$ \\
& $(0.162)$ & $(0.279)$ & $(0.019)$ & $(0.035)$ \\
DA $\times$ Scale4K & 0.055 & 0.055 & -0.012 & -0.012 \\
& $(0.240)$ & $(0.240)$ & $(0.027)$ & $(0.027)$ \\
Period & & -0.002 & & $-0.004 * * *$ \\
& & $(0.008)$ & & $(0.001)$ \\
Lottery & & -0.048 & & $-0.037 * *$ \\
& & $(0.064)$ & & $(0.009)$ \\
Lottery $\times$ DA & & -0.019 & & $0.029 * * *$ \\
& & $(0.071)$ & & $(0.009)$ \\
Lottery $\times$ LargeScale & & -0.022 & & 0.005 \\
& & $(0.071)$ & & $(0.009)$ \\
Constant & $11.172^{* * *}$ & $11.312 * * *$ & $0.116^{* * *}$ & $0.251^{* * *}$ \\
& $(0.154)$ & $(0.295)$ & $(0.017)$ & $(0.038)$ \\
\hline Observations & 3,200 & 3,200 & 3,200 & 3,200 \\
R-squared & 0.005 & 0.007 & 0.017 & 0.037 \\
\hline
\end{tabular}

Notes: Standard errors clustered at individual level, shown in parentheses. $* * * \mathrm{p}<0.01, * * \mathrm{p}<$ $0.05, * \mathrm{p}<0.1$

groups of size 40 or 4000 for each period and mechanism by drawing on human subject behavior from the $m=40$ all-human sessions. For each simulated group, students are assigned to schools based on their reported preferences. We then randomly generate 2000 groups for each human observation, and calculate the probability that a student may exhibit justified envy in the 2000 simulated matchings. The results are summarized below.

Result 10 (Stability: mechanism effect). For each given market size, the proportion of students exhibiting justified envy is significantly lower under the DA than under the Boston mechanism.

Support. In specifications (3) and (4) of Table 8, the coefficients for DA are negative and significant $(-0.076, p<0.01)$, whereas neither of the coefficients for DA $\times$ Scale40 is significant. Likewise, the coefficients for DA are negative and significant $(-0.063$ and $-0.135, p<0.01)$ in specifications (3) and (4) in Table 9, whereas neither of the coefficients for DA $\times$ Scale4K is significant.

By Result 10, we reject the null in favor of Hypothesis 5. We also observe that the none of 


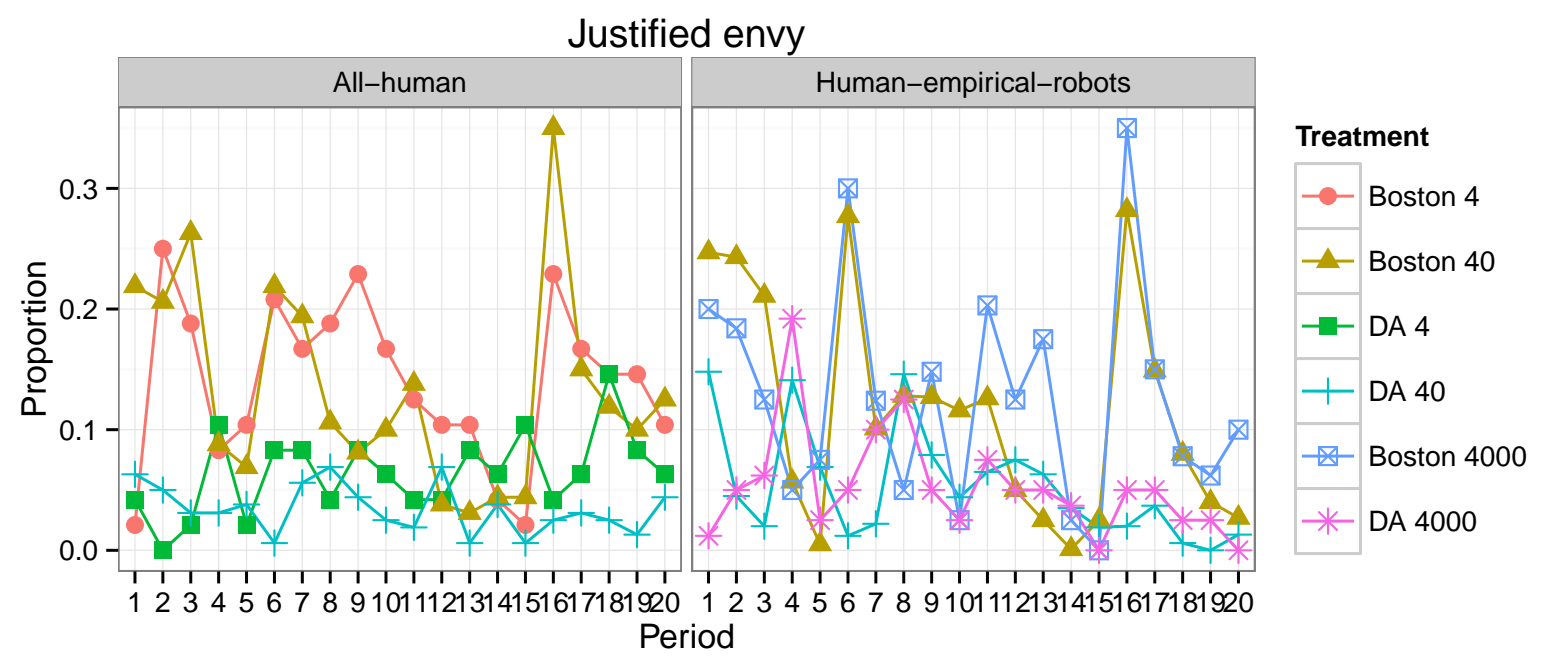

Figure 3: Justified envy in all-human (left panel) and human-vs-empirical-robots (right panel) treatments

the scale variables in Tables 8 and 9 is significant, indicating the absence of any scale effect on stability, which is consistent with the theoretical predictions. Thus, we fail to reject Hypothesis 6.

\section{Conclusion}

In practice, matching mechanisms are implemented across all size markets, from hundreds of students in course allocations, to tens of thousands in school choice, to millions in centralized college admissions markets. However, it is unclear whether market size plays a role in either participant behavior or mechanism performance. Complicating the issue is the fact that most laboratory matching experiments work with only small-scale markets.

In this paper, we use the school choice context to present a laboratory experiment investigating how matching market scale affects individual behavior and the performance of the Boston and the DA mechanisms. Specifically, we investigate the scale effect by varying the number of students per match from 4 to 40 , and then from 40 to 4,000. The results of our study reveal a significant scale effect on individual behavior when we increase the scale from 4 to 40 . Specifically, subjects become more likely to reveal their preferences truthfully under the DA mechanism but less likely to do so under the Boston mechanism. Thus, we conclude that the well-known preference manipulation gap between the DA and the Boston mechanisms in small markets increases when the size of the market increases by tenfold. Furthermore, we find that this magnification effect is due to an increased likelihood of individual players to adopt secure strategies in larger markets. Under the 
Boston mechanism, this secure strategy comes through in district school bias, while under the DA mechanism, it comes through in truthful preference revelation. However, increasing the market size to 4,000 students per match has no additional effect on individual behavior.

Examining mechanism performance at the aggregate level, we find that, while the Boston mechanism assigns more students to their first choices, the DA mechanism assigns more students to their top two choices, i.e., individually rational outcomes. Furthermore, the DA mechanism has a large and significant stability advantage over the Boston mechanism, which remains robust across all size markets. A further increase to 4,000-student per match makes no additional change on the aggregate performance of either mechanism.

In addition to our finding regarding participant behavior and mechanism performance, we contribute to the literature by using robots to create our large matching markets in a standard laboratory setting. Specifically, we group human subjects with robots whose strategies are drawn from empirical human strategies obtained from our all-human sessions. Previous experiments with robots endow the robots with either a dominant or random choice strategy. In comparison, we endow them with empirical strategies, providing a novel solution to the problems associated with conducting large group experiments in the lab.

Lastly, our findings provide insight into the ongoing theoretical debate on when market size becomes a factor in matching mechanism performance. Our results indicate that "large" might be much smaller than predicted in the theoretical literature. 
Subsequent appendices are for online publication only. 


\section{A Instructions (Translated from Chinese)}

The complete instructions for the Boston Empirical 4000 treatment (i.e., the treatment for the Boston mechanism where each human subject plays with empirical robots to form a group of 4000) are shown here. The instruction for the Boston Empirical 40 treatment are identical except for the number of players, and instructions for the Boston Truthful 4000 treatment are identical except for the subsection "Computer Programs' Strategy ..."; the instructions for the Boston Truthful 40 treatment are identical except for the number of players and the subsection "Computer Programs' Strategy..."; the instructions for the Boston All-human treatment are identical except that the number of players per group is 40 and all the players are human subjects (there is no computer program). Thus, only the different parts of the instructions for the other Boston mechanism treatments are presented here.

The instructions for the DA treatments are identical to their Boston counterparts except for the subsection "The process school admission..." and the worksheet for Review Questions \# 1 and \#11. Hence, only these two parts for DA Empirical 4000 instruction are provided here; instructions for the other DA mechanism treatments are omitted, but are available from the authors upon request.

\section{A.1 Instructions for the Boston Empirical 4000 Treatment}

\section{Instruction - BE4000}

This is an experiment in the economics of decision making. In this experiment, we simulate a procedure to allocate students to schools. The procedure, payment rules, and student allocation method are described below. The amount of money you earn will depend upon the decisions you make and on the decisions other participants make. Do not communicate with each other during the experiment. If you have questions at any point during the experiment, raise your hand and the experimenter will help you. At the end of the instructions, you will be asked to provide answers to a series of review questions. Once everyone has finished the review questions, we will go through the answers together.

\section{Procedure}

- In this experiment, you are matched with 3999 automated computer programs as a group of 4000, which will make decisions under the same situation as yours. We will describe their strategies later.

- Other than you, there is no other human participant in your group. 4000 participants (including 3999 computer programs and yourself) are classified into 4 different types, depending on 
their preferences over schools. Therefore, each group contains 1000 of each of the four different types. Your type will be shown on your computer screen when the experiment starts. Your type remains the same throughout the experiment.

- In this experiment, 4 schools are available for each group. Each school has 1000 slots.

- Your payoff amount depends on the school you are assigned to at the end of each period. Payoff amount for 4 types of students are outlined in the following table.

\begin{tabular}{l|cccc}
\hline \hline & A & B & C & D \\
\hline Payoff to Type 1 & $\underline{11}$ & 7 & 5 & 16 \\
Payoff to Type 2 & 5 & $\underline{11}$ & 7 & 16 \\
Payoff to Type 3 & 7 & 16 & $\underline{11}$ & 5 \\
Payoff to Type 4 & 5 & 16 & 7 & $\underline{11}$ \\
\hline \hline
\end{tabular}

Your district school is underlined. In other words, you reside in the underlined school district. If a school is your district school, you have the higher priority than other non-district-school students at this school.

- In this experiment, participants are defined as belonging to the following school districts:

The district school for Type 1 students is school A;

The district school for Type 2 students is school B;

The district school for Type 3 students is school C;

The district school for Type 4 students is school D.

- Your assigned school and your payoff have the following relation:

If you are Type 1 student:

You will be paid 11 points if you hold a slot of School A at the end of a period. You will be paid 7 points if you hold a slot of School B at the end of a period. You will be paid 5 points if you hold a slot of School C at the end of a period. You will be paid 16 points if you hold a slot of School D at the end of a period.

The same goes for other types.

- Just like you, computer programs will also make the decision based on the above payoffs table and whether the school is district/non-district school. 
- The experiment consists of 20 periods. In each period, you are matched with 3999 computer programs to form a group of 4000; each group contains 1000 of each of the four different types. Your earnings for each period depend on your choices as well as the choices of the 3999 other participants (computer programs) you are matched with.

- Every period, each participant (including computer programs) will rank the schools. Note that you need to rank all 4 schools in order to indicate your preferences.

- After all participants have submitted their rankings, the server will start the school placement in each group and inform each person of his/her admitted school and respective payoff. Note that your allocation in each period is independent of your allocations in the previous periods.

- Your total payoff equals the sum of your payoffs in all 20 periods. Your earnings are given in points. At the end of the experiment you will be paid based on the exchange rate:

\section{$1 \mathrm{RMB}=5$ points.}

You will be paid 5 points for answering each Review Question correctly.

In addition, you will be paid $5 \mathrm{RMB}$ for participation. Everyone will be paid in private and you are under no obligation to tell others how much you earn.

\section{Admission Method}

\section{- The priority order for each school depends on two factors:}

- Whether the student lives within the school district: the school will give higher priority to participants who live within the school district, and give low priority to participants who do not live within the school district.

- Random lottery number: the server will generate random lottery number at the beginning of every 5 periods. Each student (human or computer program) gets a unique lottery number in the range 1 4000. The smaller the lottery number is, higher the priority a student has among the district students or non-district students.

The lottery numbers for each type in each period are shown below:

\begin{tabular}{c|cccc}
\hline \hline & Periods 1-5 & Periods 6-10 & Periods11-15 & Periods 16-20 \\
\hline Type 1 & $1 \sim 1000$ & $3001 \sim 4000$ & $2001 \sim 3000$ & $1001 \sim 2000$ \\
Type 2 & $1001 \sim 2000$ & $1 \sim 1000$ & $3001 \sim 4000$ & $2001 \sim 3000$ \\
Type 3 & $2001 \sim 3000$ & $1001 \sim 2000$ & $1 \sim 1000$ & $3001 \sim 4000$ \\
Type 4 & $3001 \sim 4000$ & $2001 \sim 3000$ & $1001 \sim 2000$ & $1 \sim 1000$ \\
\hline \hline
\end{tabular}


For example, if you are student of Type1, in the periods 1 5, you will be given a random number in the range 1 1000 at the beginning of 1st period, and the number is fixed for the rest 4 periods; in the periods 6 10, you will be given a random number in the range 3001 4000 at the beginning of 6th period; the rest can be done in the same manner.

\section{- The process school admission is described by the following method:}

Step1: a. An application to the first ranked school is sent for each participant.

b. Each school accepts the student with highest priority in that school. These students and their assignments are removed from the system. The remaining applications for each respective school are rejected.

Step2: a. The rejected applications are sent to his/her second choice.

b. If a school is still vacant, then it accepts the student with the highest priority and rejects the remaining applications.

Step3: a. The application of each participant who is rejected by his/her top two choices is sent to his/her third choice.

b. If a school is still vacant, then it accepts the student with the highest priority and rejects the remaining applications.

Step4: $\quad$ Each remaining participant is assigned a slot at his/her last choice.

\section{Computer Programs' Strategy}

Computer programs will make their decisions based on the following rule:

Before this experiment, there was another similar experiment. The differences between these two experiments are:

- In this experiment, there are 3999 computer programs in a 4000-students group; in the previous experiment, all 40 participants in the group are human participants like you (not computer programs). There are 4 types of students in the previous experiment, which are the same as this experiment. But there are only 10 students of each type in the previous experiment. There are 4 schools, each with 10 slots in the previous experiment, not 1000 slots as this experiment.

- There are 80 participants in that experiment. Two 40-participants groups are randomly formed at the begining of every round. That is, each round each participant is matched with 39 different others.

Everything else, such as the student allocation method is the same in these two experiments. 
In this experiment, each period, a computer program will randomly pick one out of the two participants (not computer program) of the same type and with the corresponding lottery numbers in the previous experiment, and rank schools in the same order as her or him.

The correspondence of lottery numbers between the two experiments: the rule of generating lottery numbers in the previous experiment is similar as this experiment, which is tabulated below.

\begin{tabular}{l|cccc}
\hline \hline & Periods 1-5 & Periods 6-10 & Periods11-15 & Periods 16-20 \\
\hline Type 1 & $1 \sim 10$ & $31 \sim 40$ & $21 \sim 30$ & $11 \sim 20$ \\
Type 2 & $11 \sim 20$ & $1 \sim 10$ & $31 \sim 40$ & $21 \sim 30$ \\
Type 3 & $21 \sim 30$ & $11 \sim 20$ & $1 \sim 10$ & $31 \sim 40$ \\
Type 4 & $31 \sim 40$ & $21 \sim 30$ & $11 \sim 20$ & $1 \sim 10$ \\
\hline \hline
\end{tabular}

In each period, students of the same type, with lottery number 1 correspond to the computer programs of the same type with the lottery numbers 1 100; students with lottery number 2 correspond to the computer programs of the same type with the lottery numbers 101 200; and so forth.

For example:

A computer program is a student of Type 1; in the 2 nd round, its lottery number is 150 , which corresponds to the lottery number 2 in the previous experiment. Meanwhile in the previous experiment, two participants of Type 1 in that same round, with lottery number 2 , ranked schools in the order of D, B, C, A and A, B, C, D, respectively. Then the computer program will rank schools in the order of D, B, C, A with probability $50 \%$, and A, B, C, D with probability $50 \%$. Other computer programs of Type 1, with lottery numbers 101 200 will also rank schools based on such rule.

All computer programs in each period will follow this rule.

\section{An example:}

We will go through a simple example to illustrate how the admission method works. This example has fewer students than the actual decisions you will make. You will be asked to work out the allocation of this example for Review Question 1. There are 6 blank spaces to fill in on the computer screen. You will be given 5 points for entering each correct answer in each space.

Students and Schools: in this example, there are 6 students, 1-6, and 3 schools, A, B and C.

Student ID number: 1,2,3,4,5,6 $\quad$ Schools: A, B, C


School A is the district school of student 1 and 2; school B is the district school of student 3 and 4; school $\mathrm{C}$ is the district school of student 5 and 6.

Ranking of schools based on the lottery: Students' lottery numbers are indicated in the table below.

\begin{tabular}{c|c}
\hline \hline Student & Lottery number \\
\hline 1 & 1 \\
2 & 2 \\
3 & 3 \\
4 & 4 \\
5 & 5 \\
6 & 6 \\
\hline \hline
\end{tabular}

Submitted School Rankings:

\begin{tabular}{c|ccc}
\hline \hline Student & 1st Choice & 2nd Choice & 3rd Choice \\
\hline 1 & $\mathrm{C}$ & $\mathrm{A}$ & $\mathrm{B}$ \\
2 & $\mathrm{C}$ & $\mathrm{A}$ & $\mathrm{B}$ \\
3 & $\mathrm{~A}$ & $\mathrm{~B}$ & $\mathrm{C}$ \\
4 & $\mathrm{~A}$ & $\mathrm{~B}$ & $\mathrm{C}$ \\
5 & $\mathrm{C}$ & $\mathrm{B}$ & $\mathrm{A}$ \\
6 & $\mathrm{~A}$ & $\mathrm{C}$ & $\mathrm{B}$ \\
\hline \hline
\end{tabular}

The assignment method consists of the following steps: Please use this sheet to work out the allocation and enter it into the computer for Review Question 1.

Step 1: Each student applies to his/her first choice. If a school receives more applications than its capacity, then it accepts the applicant with the highest priority and rejects the remaining students.

\begin{tabular}{cl|c|lrr} 
Student & & School & & Accept & Reject \\
\hline $3,4,6$ & $\longrightarrow$ & $\mathrm{A}$ & $\longrightarrow$ & & \\
& & $\mathrm{B}$ & & & \\
$1,2,5$ & $\mathrm{C}$ & $\longrightarrow$ & & \\
\hline
\end{tabular}

Step 2: Each student rejected in Step 1 applies to his/her second choice. If a school still has vacancy, it accepts the applicant with the highest priority and rejects the rest. If a school is already full, it rejects all new applications.

\begin{tabular}{cl|c|lrr} 
Student & & School & & Accept & Reject \\
\hline & $\longrightarrow$ & A & $\longrightarrow$ & & \\
& B & & & \\
& $\longrightarrow$ & C & $\longrightarrow$ & &
\end{tabular}


Step 3: Each student rejected in Step 2 applies to his/her next choice.

\begin{tabular}{cl|c|lll} 
Student & & School & & Accept & Reject \\
\hline & $\longrightarrow$ & A & $\longrightarrow$ & & \\
& B & & & \\
& $\longrightarrow$ & C & $\longrightarrow$ & &
\end{tabular}

The allocation process terminates at the end of step 3.

- Please enter your answer to the computer for Review Question 1. After all have entered their answers, we will distribute an answer sheet and go through the answer together.

- Afterwards, you will be asked to answer another 10 review questions. When everyone is finished with them, we will again go through the answers together.

- Feel free to refer to the experimental instructions before you answer any question.

Answer to Review Question 1: The allocation method consists of the following steps:

Step 1: Each student applies to his/her first choice: students 3, 4 and 6 apply to A, and student 1, 2 and 5 apply to $C$.

- School A accepts the applications of students 3 and 4 , rejects student 6 , because 3 and 4 have smaller lottery numbers than 6 , and none of them are school district residents of school A.

- School C accepts the applications of student 1 and 5, rejects student 2, because 5 is a district resident, and therefore has the highest priority; between 1 and 2, 1 has a higher priority based on lottery. There is only one vacancy for school C, so it accepts student 1 and rejects student 2.

\begin{tabular}{cc|c|ccc} 
Student & & School & & Accept & Reject \\
\hline $3,4,6$ & $\longrightarrow$ & A & $\longrightarrow$ & 3,4 & 6 \\
& & B & & & \\
$1,2,5$ & $\longrightarrow$ & C & $\longrightarrow$ & 1,5 & 2 \\
\hline
\end{tabular}

Step 2: Each student rejected in Step 1 applies to his/her next choice. Student 2 applies to school A, and student 6 applies to school C. Although they both live in the school district, since school A and $\mathrm{C}$ are already full, they are both rejected. 


\begin{tabular}{cc|c|ccc} 
Student & & School & & Accept & Reject \\
\hline 2 & $\longrightarrow$ & A & $\longrightarrow$ & 3,4 & 2 \\
& B & & & \\
6 & $\longrightarrow$ & C & $\longrightarrow$ & 1,5 & 6 \\
\hline
\end{tabular}

Step 3: Each student rejected in Step 2 applies to his/her next choice. Both students 2 and 6 apply to School B.

School B accepts the applications of students 2 and 6.

\begin{tabular}{|c|c|c|c|c|c|}
\hline Student & & School & & Accept & Reject \\
\hline \multirow{3}{*}{2,6} & & $\mathrm{~A}$ & & 3,4 & \\
\hline & $\longrightarrow$ & B & $\longrightarrow$ & 2,6 & \\
\hline & & C & & 1,5 & \\
\hline
\end{tabular}

\section{Answers to Review Questions 2-11:}

2. How many participants are there in your group each period?

Answer: 4000

3. How many human participants are there in your group each period?

Answer: 1

4. True or false: Participant living in a school district has higher priority than applicants for that school who do not live in the school district.

Answer: True.

5. True or false: The priority among residents of a school district is determined by their lottery number.

Answer: True.

6. True or false: The priority among non-residents of a school district is determined by their lottery number.

Answer: True.

7. True or false: The lottery is fixed for the entire 20 periods.

Answer: False. It is randomly determined each five periods.

8. True or false: A lottery number of 1 means that I have the highest priority among the other non-resident applicants in a school.

Answer: True. 
9. True or false: If you are accepted by a school of your choice, the schools ranked below are irrelevant.

Answer: True.

10. True or false: If you are not rejected at a step, then you are accepted into that school. Answer: True.

11. True or false: The allocation is final at the end of each step.

Answer: True.

You will have 5 minutes to go over the instructions at your own pace. Feel free to earn as much as you can.

Are there any questions?

\section{A.2 Instructions for the Boston Empirical 40 Treatment}

$(\ldots .$.

Procedure

- In this experiment, you are matched with 39 automated computer programs as a group of 40, which will make decisions under the same situation as yours. We will describe their strategies later.

- Other than you, there is no other human participant in your group. 40 participants (including 39 computer programs and yourself) are classified into 4 different types, depending on their preferences over schools. Therefore, each group contains 10 of each of the four different types. Your type will be shown on your computer screen when the experiment starts. Your type remains the same throughout the experiment.

- In this experiment, 4 schools are available for each group. Each school has 10 slots.

\section{$(\ldots .$.}

- The experiment consists of 20 periods. In each period, you matched with 39 computer programs to form a group of 40; each group contains 10 of each of the four different types. 
Your earnings for each period depend on your choices as well as the choices of the 39 other participants (computer programs) you are matched with.

$(\ldots .$.

\section{Admission Method}

$(\ldots .$.

- Random lottery number: the server will generate random lottery number at the beginning of every 5 periods. Each student (human or computer program) gets a unique lottery number in the range $1 \sim 40$. The smaller the lottery number is, higher the priority a student has among the district students or non-district students.

The lottery numbers for each type in each period are shown below:

\begin{tabular}{l|cccc}
\hline \hline & Periods 1-5 & Periods 6-10 & Periods11-15 & Periods 16-20 \\
\hline Type 1 & $1 \sim 10$ & $31 \sim 40$ & $21 \sim 30$ & $11 \sim 20$ \\
Type 2 & $11 \sim 20$ & $1 \sim 10$ & $31 \sim 40$ & $21 \sim 30$ \\
Type 3 & $21 \sim 30$ & $11 \sim 20$ & $1 \sim 10$ & $31 \sim 40$ \\
Type 4 & $31 \sim 40$ & $21 \sim 30$ & $11 \sim 20$ & $1 \sim 10$ \\
\hline \hline
\end{tabular}

For example, if you are student of Type1, in the periods 1 5, you will be given a random number in the range 1 10 at the beginning of 1st period, and the number is fixed for the rest 4 periods; in the periods $6 \sim 10$, you will be given a random number in the range $31 \sim 40$ at the beginning of 6th period; the rest can be done in the same manner.

$(\ldots .$.

\section{Computer Programs' Strategy}

Computer programs will make their decisions based on the following rule:

Before this experiment, there was another similar experiment. The differences between these two experiments are:

- In this experiment, there are 39 computer programs in a 40-students group; in the previous experiment, all 40 participants in the group are human participants like you (not computer programs). 
- There are 80 participants in that experiment. Two 40-participants groups are randomly formed at the begining of every round. That is, each round each participant is matched with 39 different others.

Everything else, such as the types of students, the payoff for being admitted to each school, the student allocation method, and the arrangement of lottery numbers are the same in these two experiments.

In this experiment, each period, a computer program will randomly pick one out of the two participants (not computer program) of the same type and with the same lottery number in the previous experiment, and rank schools in the same order as her or him.

For example:

A computer program is a student of Type 1: in the 2 nd round, its lottery number is 2 . Meanwhile in the previous experiment, two participants of Type 1 in that same round, with lottery number 2 , ranked schools in the order of D, B, C, A and A, B, C, D, respectively. Then the computer program will rank schools in the order of $\mathrm{D}, \mathrm{B}, \mathrm{C}, \mathrm{A}$ with probability $50 \%$, and $\mathrm{A}, \mathrm{B}, \mathrm{C}, \mathrm{D}$ with probability $50 \%$.

All computer programs in each period will follow this rule.

$(\ldots .$.

Answers to Review Questions 2-11:

2. How many participants are there in your group each period? Answer: 40

$(\ldots .$.

\section{A.3 Instructions for the Boston Truthful 4000 Treatment}<smiles>[Te]</smiles>

\section{Computer Programs' Strategy}

Computer programs will make their decisions based on the following rule: 
Computer programs will always rank schools based on their true preference orders.

For example, a computer program is a student of Type 2, therefore its preference order of schools is $(\mathrm{D}, \mathrm{B}, \mathrm{C}, \mathrm{A})$. It then will also rank school $(\mathrm{D}, \mathrm{B}, \mathrm{C}, \mathrm{A})$ on his list.

$(\ldots .$.

\section{A.4 Instructions for the Boston Truthful 40 Treatment}

$(\ldots .$.

Procedure

- In this experiment, you are matched with 39 automated computer programs as a group of 40 , which will make decisions under the same situation as yours. We will describe their strategies later.

- Other than you, there is no other human participant in your group. 40 participants (including 39 computer programs and yourself) are classified into 4 different types, depending on their preferences over schools. Therefore, each group contains 10 of each of the four different types. Your type will be shown on your computer screen when the experiment starts. Your type remains the same throughout the experiment.

- In this experiment, 4 schools are available for each group. Each school has 10 slots.<smiles>[Li][V]</smiles>

- The experiment consists of 20 periods. In each period, you matched with 39 computer programs to form a group of 40; each group contains 10 of each of the four different types. Your earnings for each period depend on your choices as well as the choices of the 39 other participants (computer programs) you are matched with.

$(\ldots .$.

\section{Admission Method}

$(\ldots .$. 
- Random lottery number: the server will generate random lottery number at the beginning of every 5 periods. Each student (human or computer program) gets a unique lottery number in the range $1 \sim 40$. The smaller the lottery number is, higher the priority a student has among the district students or non-district students.

The lottery numbers for each type in each period are shown below:

\begin{tabular}{l|cccc}
\hline \hline & Periods 1-5 & Periods 6-10 & Periods11-15 & Periods 16-20 \\
\hline Type 1 & $1 \sim 10$ & $31 \sim 40$ & $21 \sim 30$ & $11 \sim 20$ \\
Type 2 & $11 \sim 20$ & $1 \sim 10$ & $31 \sim 40$ & $21 \sim 30$ \\
Type 3 & $21 \sim 30$ & $11 \sim 20$ & $1 \sim 10$ & $31 \sim 40$ \\
Type 4 & $31 \sim 40$ & $21 \sim 30$ & $11 \sim 20$ & $1 \sim 10$ \\
\hline \hline
\end{tabular}

For example, if you are student of Type1, in the periods 1 5, you will be given a random number in the range 1 10 at the beginning of 1 st period, and the number is fixed for the rest 4 periods; in the periods $6 \sim 10$, you will be given a random number in the range $31 \sim 40$ at the beginning of 6th period; the rest can be done in the same manner.

$(\ldots .$.

\section{Computer Programs' Strategy}

Computer programs will make their decisions based on the following rule:

Computer programs will always rank schools based on their true preference orders.

For example, a computer program is a student of Type 2, therefore its preference order of schools is $(\mathrm{D}, \mathrm{B}, \mathrm{C}, \mathrm{A})$. It then will also rank school $(\mathrm{D}, \mathrm{B}, \mathrm{C}, \mathrm{A})$ on his list.

$(\ldots .$.

\section{Answers to Review Questions 2-11:}

2. How many participants are there in your group each period? Answer: 40

$(\ldots .$. 


\section{A.5 Instructions for the Boston All-human Treatment}

\section{$(\ldots .$.}

\section{Procedure}

- There are 80 participants of 4 different types in this experiment. Your type will be shown on your computer screen after the experiment starts. Your type remains the same throughout the experiment.

- You will be randomly matched with other 39 participants into groups of 40 at the beginning of each period. 4 schools are available for each group. Each school has 10 slots.

\section{$(\ldots .$.}

- The experiment consists of 20 periods. In each period, you are randomly matched with other 39 participants to form a group of 40; each group contains 10 of each of the four different types. Your earnings for each period depend on your choices as well as the choices of the 39 other participants you are matched with.

- Every period, each participant will rank the schools. Note that you need to rank all 4 schools in order to indicate your preferences.

$(\ldots .$.

\section{Admission Method}

$(\ldots .$.

- Random lottery number: the server will generate random lottery number at the beginning of every 5 periods. Each student gets a unique lottery number in the range 1 40. The smaller the lottery number is, higher the priority a student has among the district students or nondistrict students.

The lottery numbers for each type in each period are shown below: 


\begin{tabular}{l|cccc}
\hline \hline & Periods 1-5 & Periods 6-10 & Periods11-15 & Periods 16-20 \\
\hline Type 1 & $1 \sim 10$ & $31 \sim 40$ & $21 \sim 30$ & $11 \sim 20$ \\
Type 2 & $11 \sim 20$ & $1 \sim 10$ & $31 \sim 40$ & $21 \sim 30$ \\
Type 3 & $21 \sim 30$ & $11 \sim 20$ & $1 \sim 10$ & $31 \sim 40$ \\
Type 4 & $31 \sim 40$ & $21 \sim 30$ & $11 \sim 20$ & $1 \sim 10$ \\
\hline \hline
\end{tabular}

For example, if you are student of Type1, in the periods 1 5, you will be given a random number in the range 1 10 at the beginning of 1st period, and the number is fixed for the rest 4 periods; in the periods $6 \sim 10$, you will be given a random number in the range $31 \sim 40$ at the beginning of 6th period; the rest can be done in the same manner.

$(\ldots .$.

[There is no subsection "Compter Programs' Strategy..." in the instructions for the Boston All-human Treatment.]

$(\ldots .$.

\section{Answers to Review Questions 2-11:}

2. How many participants are there in your group each period?

Answer: 40

3. True or false: You will be matched with the same 39 participants each period. Answer: False. You are randomly re-matched with 39 other participants each period.

$(\ldots .$.

\section{A.6 Instructions for the Deferred Acceptance Empirical 4000 Treatment}

$(\ldots .$.

- The process school admission is described by the following method: 
Step1: Each student applies to his first choice school. For each school, up to the number of applicants that does not exceed its capacity, who have the highest priority for this school, are tentatively accepted. The remaining applicants are rejected.

Step2: Each student rejected from a school at step 1 applies to his next favorite acceptable school. For each school, it pools together the new applicants and the students that have already been tentatively placed, and tentatively accepts those who have the highest priority within its capacity. The remaining applicants are rejected.

The process continues until there is no student being rejected. Then all tentative acceptances become permanent.

$(\ldots .$.

\section{An example:}

$(\ldots .$.

Submitted School Rankings:

\begin{tabular}{c|ccc}
\hline \hline Student & 1st Choice & 2nd Choice & 3rd Choice \\
\hline 1 & $\mathrm{C}$ & $\mathrm{B}$ & $\mathrm{A}$ \\
2 & $\mathrm{C}$ & $\mathrm{A}$ & $\mathrm{B}$ \\
3 & $\mathrm{~A}$ & $\mathrm{~B}$ & $\mathrm{C}$ \\
4 & $\mathrm{~A}$ & $\mathrm{~B}$ & $\mathrm{C}$ \\
5 & $\mathrm{C}$ & $\mathrm{B}$ & $\mathrm{A}$ \\
6 & $\mathrm{~A}$ & $\mathrm{C}$ & $\mathrm{B}$ \\
\hline \hline
\end{tabular}

The assignment method consists of the following steps: Please use this sheet to work out the allocation and enter it into the computer for Review Question 1.

Step 1: Each student applies to his/her first choice. If a school receives more applications than its capacity, then it temporarily holds the applications with the highest priority within its capacity and rejects the remaining students.

\begin{tabular}{cl|c|ll} 
Student & & School & & Hold Reject \\
\hline $3,4,6 \longrightarrow$ & A & $\longrightarrow$ & & \\
& & B & & \\
$1,2,5 \longrightarrow$ & C & $\longrightarrow$ & & \\
\hline
\end{tabular}

Step 2: Each student rejected in Step 1 applies to his/her next choice. When a school receives new applications, these applications are considered together with the application on hold for that 
school. Among the new applications and those on hold, the one with the highest priority is on hold within the school's capacity, while the rest are rejected.

\begin{tabular}{ll|c|lll} 
Hold New Applicants & & School & & Hold & Reject \\
\hline & $\longrightarrow$ & A & $\longrightarrow$ & & \\
& $\longrightarrow$ & B & $\longrightarrow$ & & \\
& $\longrightarrow$ & C & $\longrightarrow$ & & \\
\hline
\end{tabular}

Step 3: Each student rejected in Step 2 applies to his/her next choice. No one is rejected at this step. All students on hold are accepted.

\begin{tabular}{ll|c|lll} 
Hold New Applicants & & School & & Accept & Reject \\
\hline & $\longrightarrow$ & A & $\longrightarrow$ & & \\
& $\longrightarrow$ & B & $\longrightarrow$ & & \\
& $\longrightarrow$ & C & $\longrightarrow$ & & \\
\hline
\end{tabular}

The allocation process terminates at the end of step 3.

- Please enter your answer to the computer for Review Question 1. After all have entered their answers, we will distribute an answer sheet and go through the answer together.

- Afterwards, you will be asked to answer another 10 review questions. When everyone is finished with them, we will again go through the answers together.

- Feel free to refer to the experimental instructions before you answer any question.

Answer to Review Question 1: The allocation method consists of the following steps:

Step 1: Each student applies to his/her first choice: students 3, 4 and 6 apply to A, and student 1, 2 and 5 apply to $\mathrm{C}$.

- School A accepts the applications of students 3 and 4, rejects student 6, because 3 and 4 have smaller lottery numbers than 6 , and none of them are school district residents of school A.

- School $\mathrm{C}$ accepts the applications of student 1 and 5 , rejects student 2, because 5 is a district resident, and therefore has the highest priority; between 1 and 2, 1 has a higher priority based on lottery. There is only one vacancy for school $\mathrm{C}$, so it accepts student 1 and rejects student 2. 


\begin{tabular}{cc|c|ccc} 
Student & & School & & Hold & Reject \\
\hline $3,4,6$ & $\longrightarrow$ & A & $\longrightarrow$ & 3,4 & 6 \\
& & B & & & \\
$1,2,5$ & $\longrightarrow$ & C & $\longrightarrow$ & 1,5 & 2 \\
\hline
\end{tabular}

Step 2: Each student rejected in Step 1 applies to his/her next choice. Student 2 applies to school A, and student 6 applies to school B.

- School A considers the application of student 2 together with the applications of students 3 and 4 which were on hold. Student 2 is a resident in that school district, and student 3 has a smaller lottery number, therefore school A holds students 2 and 3's applications, and rejects student 4.

- Similarly, school C holds students 5 and 6's applications, and rejects student 1.

\begin{tabular}{ccc|c|lcc} 
Hold & New Applicants & & School & & Hold & Reject \\
\hline 3,4 & 2 & $\longrightarrow$ & A & $\longrightarrow$ & 2,3 & 4 \\
& & & B & & & \\
1,5 & 6 & $\longrightarrow$ & C & $\longrightarrow$ & 5,6 & 1 \\
\hline
\end{tabular}

Step 3: Each student rejected in Step 2 applies to his/her next choice. Student 1 and 4 both apply to School B in the next choice.

School B holds the applications of student 1 and 4.

Since no student is rejected after this step, all tentative holdings become permanent acceptance.

\begin{tabular}{|c|c|c|c|c|c|c|}
\hline Hold & New Applicants & & School & & Accept & Reject \\
\hline 2,3 & & & $\mathrm{~A}$ & & 2,3 & \\
\hline & 1,4 & $\longrightarrow$ & B & $\longrightarrow$ & 1,4 & \\
\hline 5,6 & & & $\mathrm{C}$ & & 5,6 & \\
\hline
\end{tabular}

\section{Answers to Review Questions 2-11:}<smiles>[Li]</smiles>

11. True or false: The allocation is final at the end of each step.

Answer: False. The allocation at the end of every step is temporary except the final step. 


\section{Appendix B. Additional Tables and Figures}

This appendix presents additional tables and figures, organized by section.

\section{B.1. Experimental Design}

Table 10: Priority Queue

\begin{tabular}{c|ccccc}
\hline & Scale & Type 1 & Type 2 & Type 3 & Type 4 \\
\hline \multirow{3}{*}{ Block 1: periods 1-5 } & 4 students & 1 & 2 & 3 & 4 \\
& 40 students & $1 \sim 10$ & $11 \sim 20$ & $21 \sim 30$ & $31 \sim 40$ \\
& 4000 students & $1 \sim 1000$ & $1001 \sim 2000$ & $2001 \sim 3000$ & $3001 \sim 4000$ \\
\hline \multirow{3}{*}{ Block 2: periods 6-10 } & 4 students & 4 & 1 & 2 & 3 \\
& 40 students & $31 \sim 40$ & $1 \sim 10$ & $11 \sim 20$ & $21 \sim 30$ \\
& 4000 students & $3001 \sim 4000$ & $1 \sim 1000$ & $1001 \sim 2000$ & $2001 \sim 3000$ \\
\hline \multirow{3}{*}{ Block 3: periods 11-15 } & 4 students & 3 & 4 & 1 & 2 \\
& 40 students & $21 \sim 30$ & $31 \sim 40$ & $1 \sim 10$ & $11 \sim 20$ \\
& 4000 students & $2001 \sim 3000$ & $3001 \sim 4000$ & $1 \sim 1000$ & $1001 \sim 2000$ \\
\hline \multirow{2}{*}{ Block 4: periods 16-20 } & 4 students & 2 & 3 & 4 & 1 \\
& 40 students & $11 \sim 20$ & $21 \sim 30$ & $31 \sim 40$ & $1 \sim 10$ \\
& 4000 students & $1001 \sim 2000$ & $2001 \sim 3000$ & $3001 \sim 4000$ & $1 \sim 1000$ \\
\hline
\end{tabular}

\section{B.2. Results - Individual Behavior}

Table 11: Proportion of truthful preference revelation and district school bias

\begin{tabular}{|c|c|c|c|c|c|c|c|c|}
\hline \multicolumn{9}{|c|}{ All-human treatments } \\
\hline & \multicolumn{4}{|c|}{ Truthful Preference Revelation } & \multicolumn{4}{|c|}{ District School Bias } \\
\hline & BOS-4 & DA-4 & BOS-40 & DA-40 & BOS-4 & DA-4 & BOS-40 & DA-40 \\
\hline Session 1 & 0.421 & 0.696 & 0.353 & 0.644 & 0.550 & 0.183 & 0.607 & 0.259 \\
\hline Session2 & 0.483 & 0.596 & 0.409 & 0.693 & 0.375 & 0.271 & 0.568 & 0.261 \\
\hline Session 3 & 0.492 & 0.554 & & & 0.496 & 0.204 & & \\
\hline Session4 & 0.463 & 0.567 & & & 0.442 & 0.338 & & \\
\hline Average & 0.465 & 0.603 & 0.381 & 0.668 & 0.466 & 0.249 & 0.588 & 0.260 \\
\hline \multicolumn{9}{|c|}{ Human-vs-empirical-robot treatments } \\
\hline & \multicolumn{4}{|c|}{ Truthful Preference Revelation } & \multicolumn{4}{|c|}{ District School Bias } \\
\hline & BOS-40 & DA-40 & BOS-4K & $\mathrm{DA}-4 \mathrm{~K}$ & BOS-40 & DA-40 & BOS-4K & $\mathrm{DA}-4 \mathrm{~K}$ \\
\hline Session 1 & 0.419 & 0.602 & 0.498 & 0.708 & 0.538 & 0.283 & 0.460 & 0.216 \\
\hline Session 2 & 0.487 & 0.629 & 0.368 & 0.614 & 0.476 & 0.247 & 0.585 & 0.212 \\
\hline Average & 0.453 & 0.616 & 0.433 & 0.661 & 0.507 & 0.265 & 0.523 & 0.214 \\
\hline
\end{tabular}


We present two additional tables for individual behavior. Table 11 presents the proportion of truthful preference revelation and district school bias at the session level.

Table 12: K-S test for all-human 40-student and human-vs-39-empirical-robots sessions

\begin{tabular}{ccccccccc}
\hline \multicolumn{2}{c}{ Boston-40 $\left(H_{a}:\right.$ All human $\neq$ Empirical robot $)$} & & \multicolumn{3}{c}{ DA-40 } & \multicolumn{3}{c}{$H_{a}:$ All human $\neq$ Empirical robot $)$} \\
\cline { 1 - 4 } \cline { 6 - 8 } Period & P-value & Period & P-value & & Period & P-value & Period & P-value \\
\hline 1 & 0.218 & 11 & 0.914 & & 1 & 0.078 & 11 & 0.627 \\
2 & 0.997 & 12 & 0.814 & & 2 & 0.254 & 12 & 0.989 \\
3 & 0.868 & 13 & 0.868 & & 3 & 0.949 & 13 & 0.691 \\
4 & 0.914 & 14 & 0.997 & & 4 & 0.563 & 14 & 0.974 \\
5 & 0.997 & 15 & 0.949 & & 5 & 0.754 & 15 & 0.814 \\
6 & 0.389 & 16 & 0.989 & & 6 & 1 & 16 & 0.691 \\
7 & 1 & 17 & 0.868 & & 7 & 0.868 & 17 & 0.627 \\
8 & 0.949 & 18 & 0.627 & & 8 & 0.997 & 18 & 0.218 \\
9 & 0.502 & 19 & 0.914 & & 9 & 1 & 19 & 0.814 \\
10 & 0.295 & 20 & 0.949 & & 10 & 0.914 & 20 & 0.989 \\
\hline
\end{tabular}

Notes: $p$-values calculated for $H_{a}:$ All human $\neq$ Empirical robot, with K-S test at the individual level.

Table 12 presents the results of the Kolmogorov-Smirnov tests of the equality of distributions of strategies between the all-human 40-student treatment and the corresponding human-vs-39empirical-robots treatment, period by period. We do not pool across all periods because of the interdependency of strategies across periods. 


\section{B.3. Results - Aggregate Performance}

Figures 4 and 5 present the first-choice accommodation rate for the all-human and the humanvs-empirical-robots treatments, respectively, comparing the proportion of subjects receiving their reported (top panel) and true first choices. We see that a greater proportion receive their reported top choice under the Boston mechanism, but the gap between the two mechanisms is much smaller when examining subjects' true top choices.

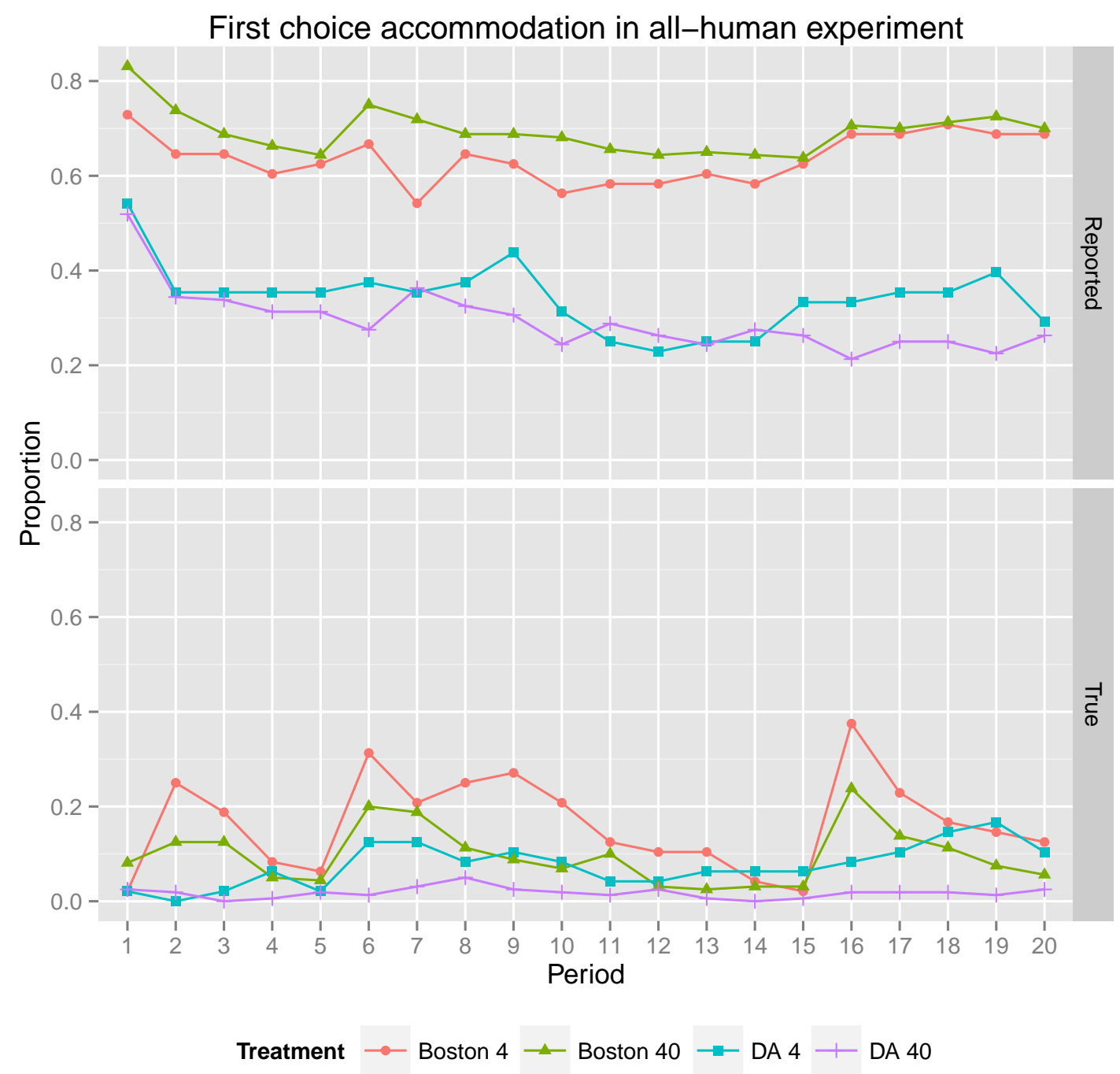

Figure 4: First choice accommodation in all-human experiment

Figure 6 presents the proportion of individually rational allocations in each treatment. 
First choice accommodation in human-empirical-robots experiment

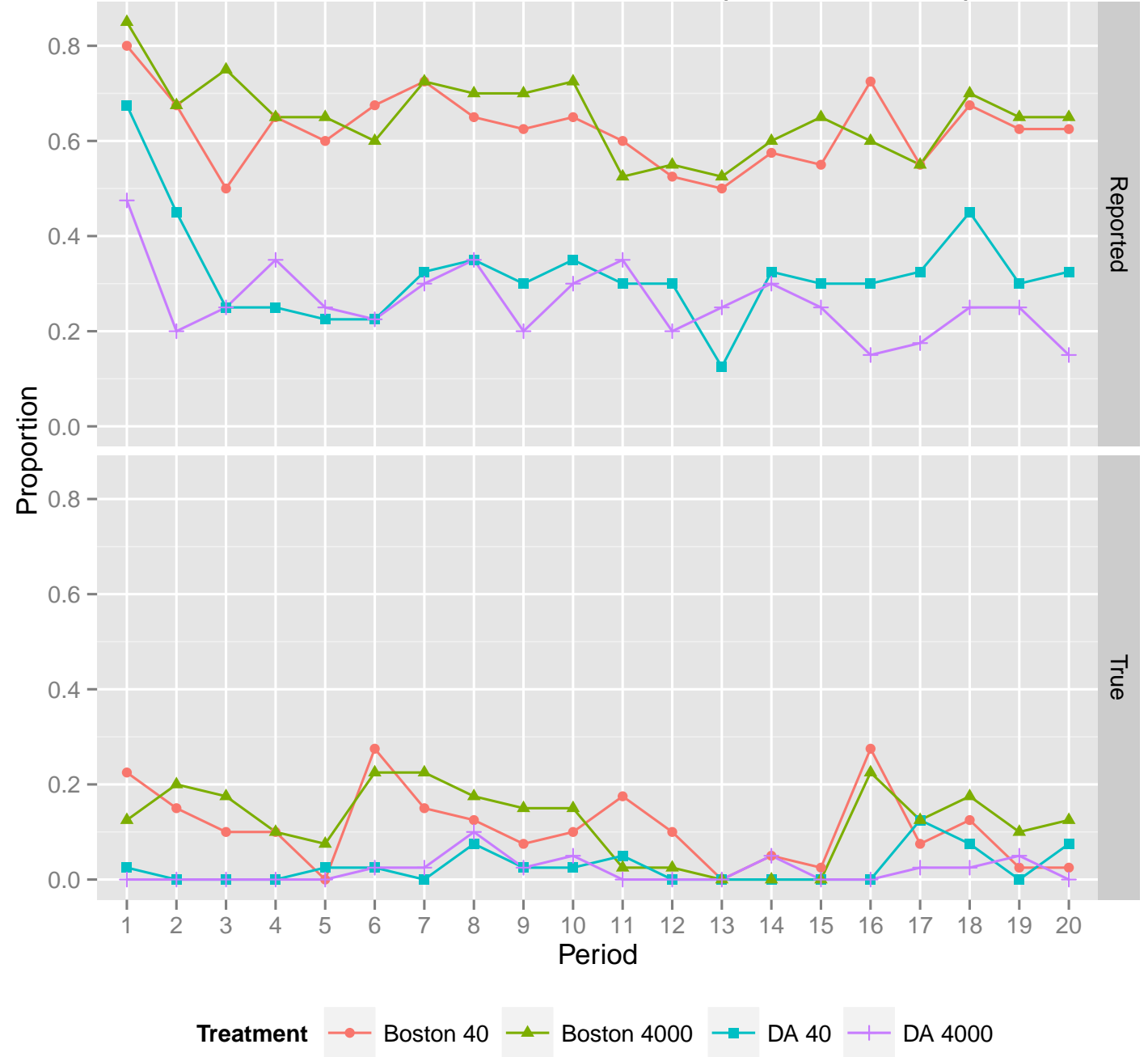

Figure 5: First choice accommodation in human-vs-empirical-robots experiment 

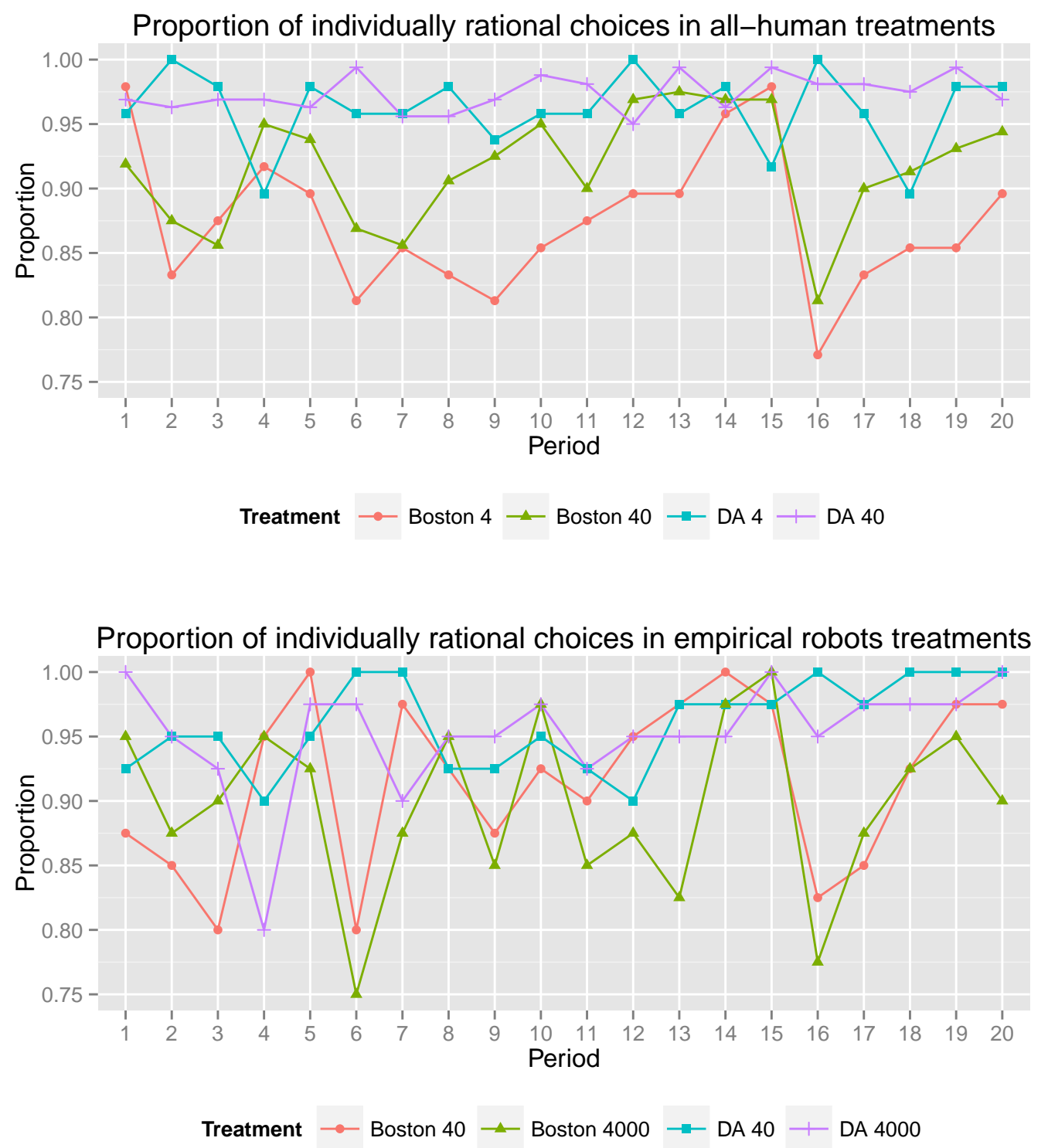

Figure 6: Individual rationality in all-human (upper panel) and human-vs-empirical-robots (lower panel) treatments 
Payoff-based efficiency in all-human experiment
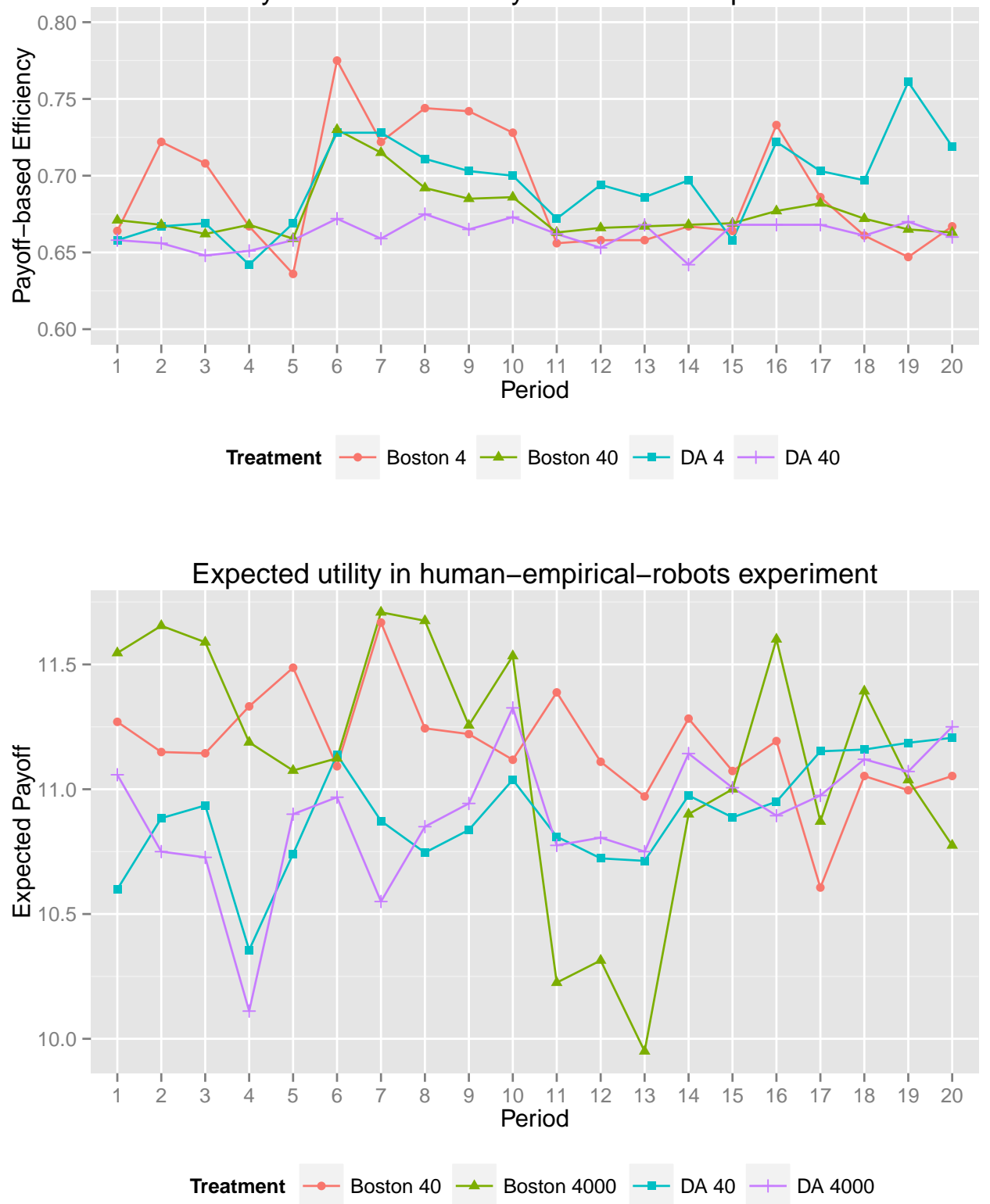

Figure 7: Payoff-based efficiency in all-human treatments (upper panel) and expected payoff of human players in human-vs-empirical-robots treatments (lower panel) 


\section{Appendix C. Best Response Analysis}

In this section, we investigate whether students' strategies are the best responses under different mechanisms with different market sizes. We start by examining the human-vs-truthful-robots treatment, where there is no strategic uncertainty regarding other players' behavior.

\section{C.1. Human-vs-truthful-robots experiment}

Using a human-robot design allows us to control robots' strategies and thus study the nature of subjects' responses. In our experiment, robots are designed to always reveal their true preferences. This, in the school choice context, is considered a "naïve" strategy (Ergin and Sönmez, 2006). Table 13 presents the best response strategies for each type in each lottery block under the Boston mechanism.

Table 13: Best Responses in the Truthful-robot Boston Treatment

\begin{tabular}{l|cccc}
\hline & Type 1 & Type 2 & Type 3 & Type 4 \\
\hline Block 1: & T1 & D & T1 & D \\
Block 2: & T2 or D & T1 & T1 & D \\
Block 3: & T1 & D & T1 & D \\
Block 4: & T1 & D & T2 or D & T1 \\
\hline
\end{tabular}

Note: $\mathrm{T} 1=$ Truthful first choice revelation; $\mathrm{T} 2=$ Truthful revelation of the first two choices; $\mathrm{D}=$ District school bias

Under the DA mechanism, when human players are matched with truthful robots, all strategies yield the same outcome. Therefore, there is no single best response. However, significantly more than $25 \%$ (if chosen by random) of first choices are truthful (Chi-square goodness of fit test against multinomial distribution, $p<0.01$ ).

Under the Boston mechanism, the situation becomes more interesting. We first establish the following definitions:

- Best response truth-telling: revealing the true preference as the best response.

- Naïve truth-telling: revealing the true preference when district bias is the best response.

- Justified district bias: having district bias when it is the best response.

- Unjustified district bias: having district bias when truth telling is a better option. 
Table 14: Breakdown of strategies in the Truthful Boston treatments

\begin{tabular}{ccc}
\hline & Small Scale & Large Scale \\
\hline Best response & 0.884 & 0.921 \\
\hline BR truth-telling & 0.465 & 0.505 \\
Justified DB & 0.419 & 0.416 \\
\hline Non-best response & 0.102 & 0.063 \\
\hline Naive truth-telling & 0.023 & 0.031 \\
Unjustified DB & 0.079 & 0.031 \\
\hline
\end{tabular}

Table 14 summarizes the proportion of best responses as well as the proportion of strategies that could be classified as each of the four types listed above.

Note that the proportion of best responses is probably overestimated, as naïve truth-telling cannot be discarded when truth-telling is a best response strategy. Furthermore, justified district bias in a certain period is unlikely to be viewed as a best response if subjects still exhibit district bias in the later periods when it is no longer a best response (such as students of Type 4 in block 4).

As a result, we need to refine what constitutes a best response: in accordance with behavioral consistency, the best response in a given period is considered only when a subject always bestresponds starting that period. If a subject best responds in one period, she should best respond in subsequent periods. Although this refined best response definition does not allow for any mistake or learning by trial and error when best response strategies switch, it may provide greater insight in our analysis.

Figure 8 presents the proportion of refined best responses over time, interpreted as the proportion of subjects who always best-respond to the environment starting that period. It is interesting to observe that this behavior increases sharply in the first three periods and then slows its growth until the last period. In the last period, $92.5 \%$ of subjects play best responses, while $42.5 \%$ of subjects play their best responses from Period 1.

In contrast, the proportion of truth-telling shown in Figure 9 indicates no learning over periods. While there is no indication of learning, Figure 8 better captures human participants' likelihood of giving a best response in such environments.

\section{C.2. All-human and human-vs-empirical-robots experiment}

Using the definition and concepts developed in the human-vs-truthful-robots experiment, we apply the same method to the other two sets of treatments. This time, however, players do not know 


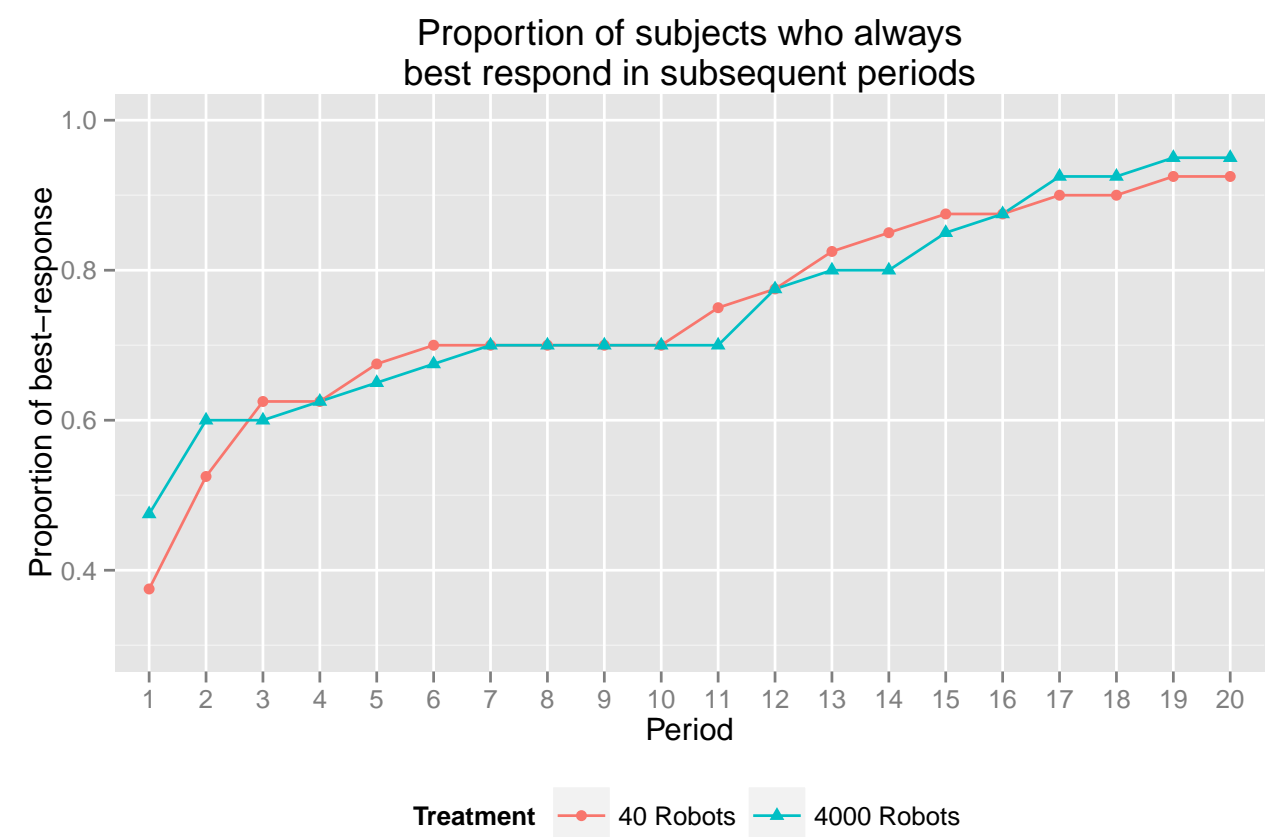

Figure 8: Proportion of subjects who always best respond in subsequent periods

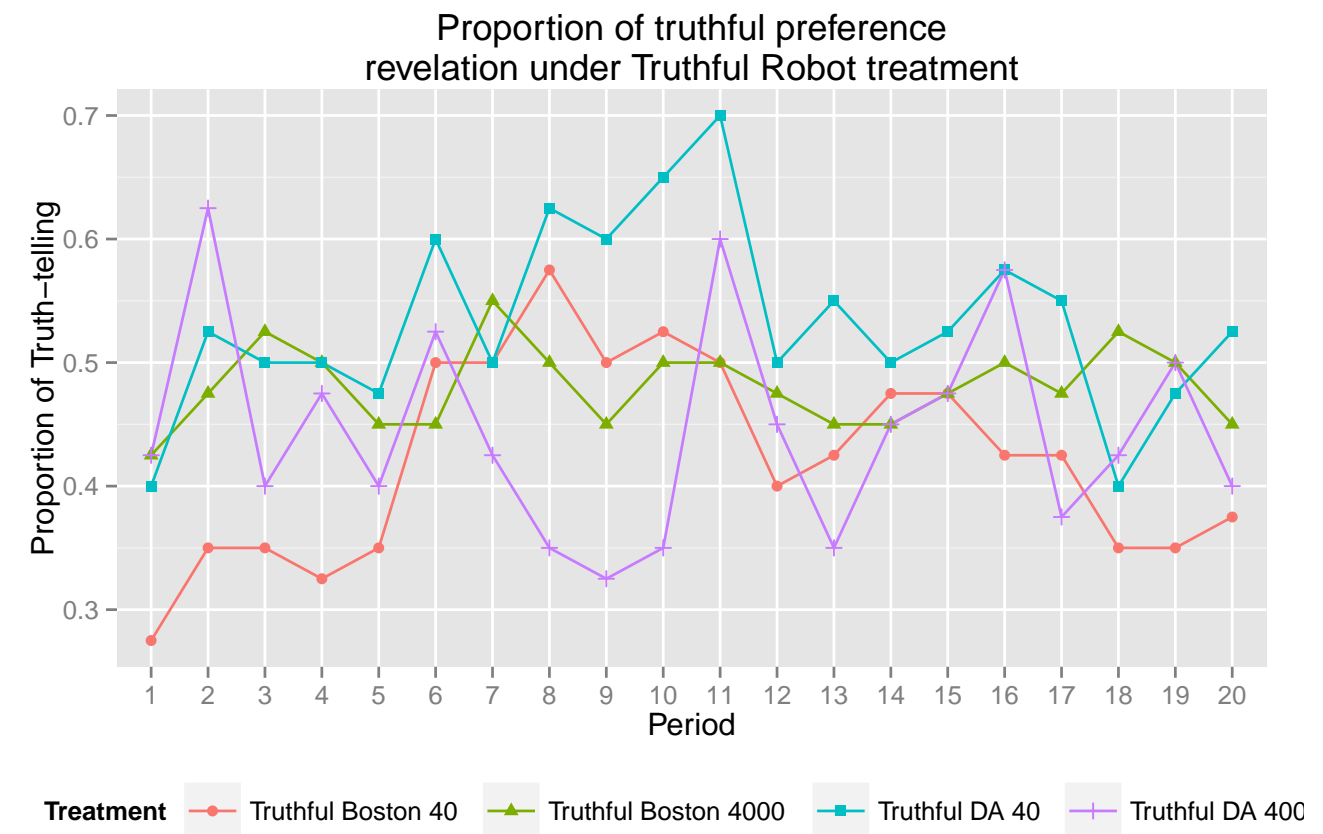

Figure 9: Proportion of truthful preference revelation under Truthful Robot treatment 
others' strategies when they decide theirs. To capture this scenario, we implement counterfactual simulations using a strategy of either truth-telling or district bias. For the all-human treatments, for each observation, we control the strategies of all other players in the group, calculate the possible payoffs under truth-telling and district bias respectively, and compare them with what that player actually earned. For the human-vs treatments, we again recreate 2000 groups for each human observation, calculate the expected payoff under truth-telling and district bias respectively, and compare them with what that player actually earned. Figures 10 and 11 present the proportions of players who best respond to other players' strategies as well as exhibit either naive truth-telling or unjustified district bias. Figures 12 and 13 present the proportions of players who always best respond since a certain period.

Result 11 (Best responses). More students play best response strategies under the DA than under Boston, and more students play best response strategies under large scale than under smaller scale market conditions.

Support. In specification (1) and (4) of Table A.6, the marginal effect of the LargeScale dummy is $0.034(p<0.05)$, and the marginal effect of DA is $0.115(p<0.01)$. In specification (4), the marginal effect of LargeScale is 0.084. ( $p<0.01)$, and the marginal effect of DA is 0.104 $(p<0.01)$. There is not interaction effect.

When we look at the set of subjects who do not play best response strategies, we can see that, for the all-human treatments, the DA significantly reduces the proportion of subjects who exhibit district bias when it is not a best response strategy $(-7.1 \%, p<0.05)$. On the other hand, when playing against robots, subjects are less like to reveal their true preferences when it is not in their interest to do so under Boston $(-10 \%, p<0.01)$.

Finally, Figures 12 and 13, and Table 16 confirm the above result when we look at the convergence over time as a result of subjects learning to play best response strategies. 


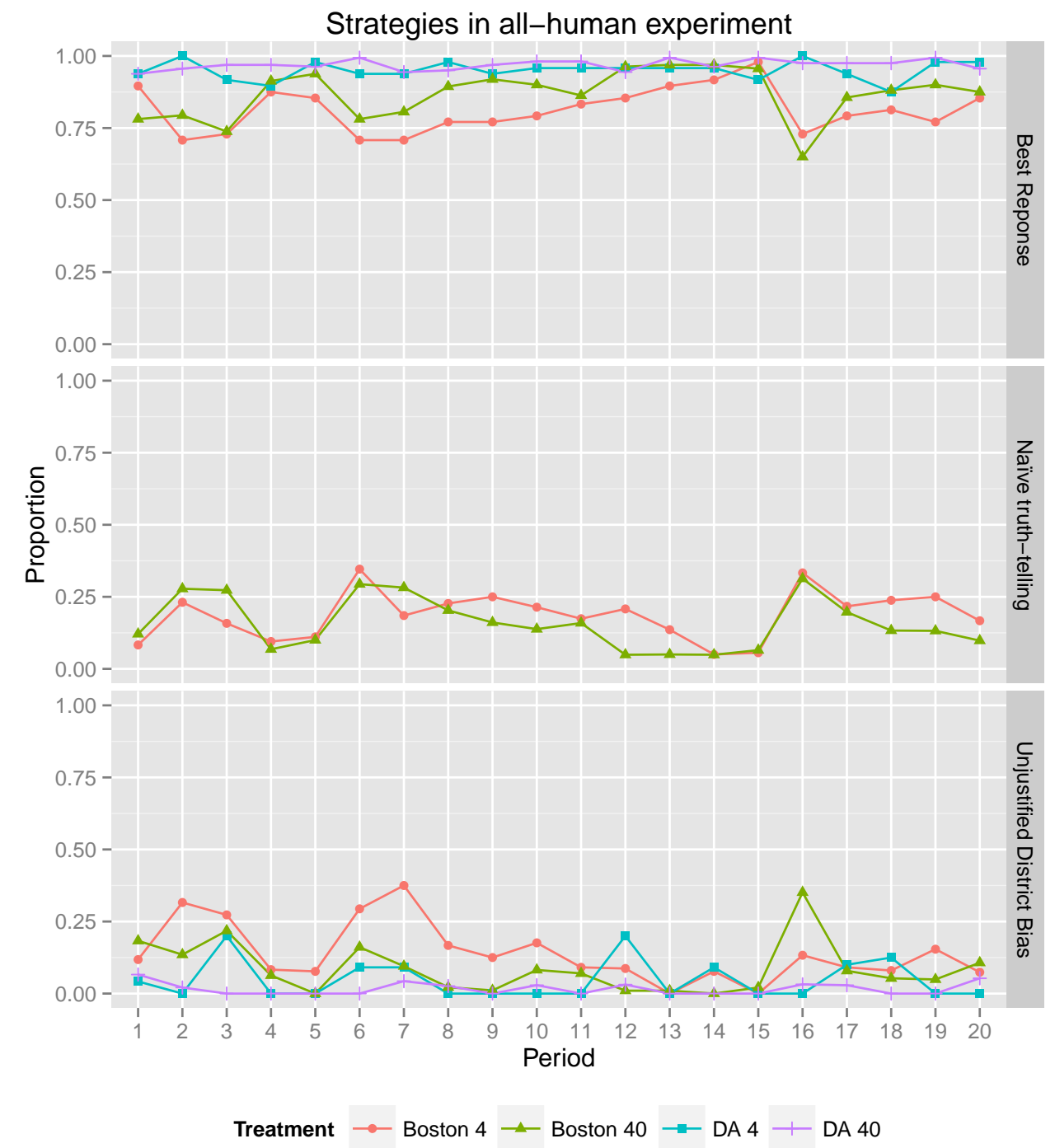

Figure 10: Best responses and other strategies in all-human experiment 


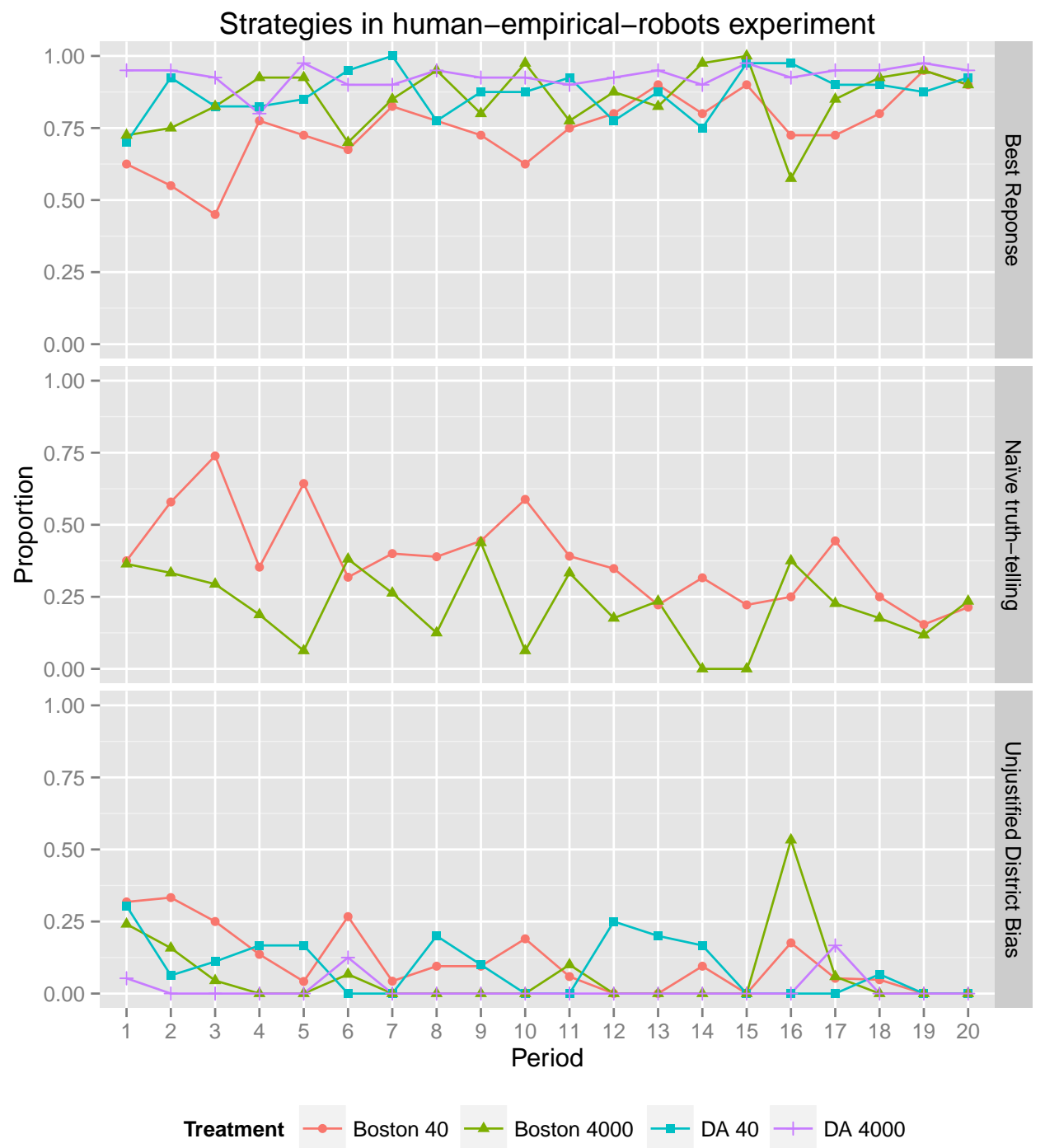

Figure 11: Best responses and other strategies in human-empirical-robots experiment 


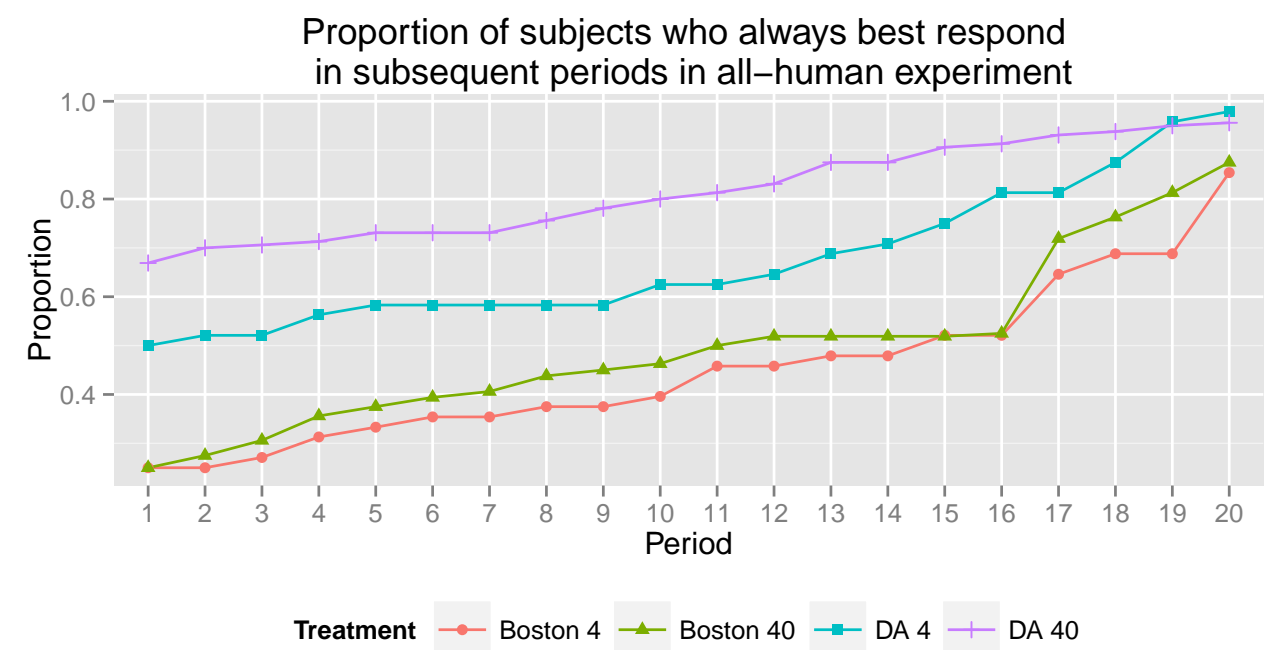

Figure 12: Proportion of subjects who always best respond in subsequent periods in all-human experiment

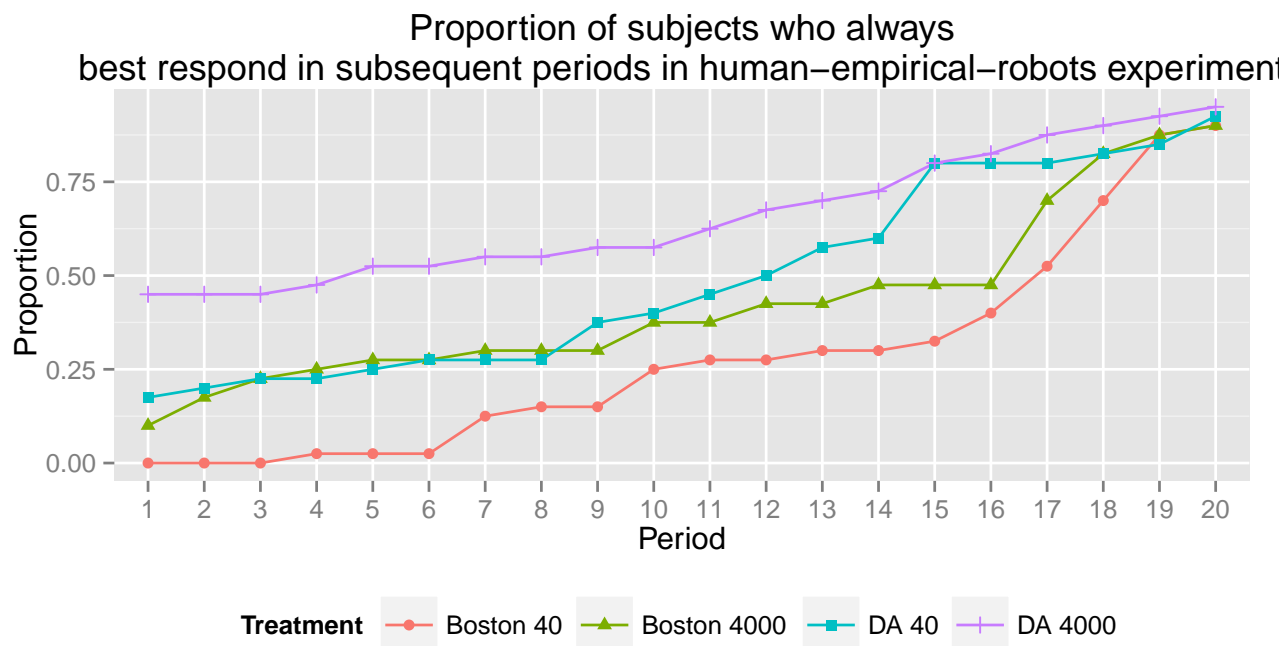

Figure 13: Proportion of subjects who always best respond in subsequent periods in humanempirical-robots experiment 
Table 15: Strategies in all-human and human-vs-empirical-robots treatments: probit regressions

\begin{tabular}{|c|c|c|c|c|c|c|}
\hline \multirow{2}{*}{ Treatment } & \multicolumn{3}{|c|}{ All-human } & \multicolumn{3}{|c|}{ Empirical-robots } \\
\hline & (1) & (2) & (3) & (4) & (5) & (6) \\
\hline Dependent & Best & Naïve & Unjustified & Best & Naïve & Unjustified \\
\hline Variables & Response & Truth-telling & District Bias & Response & Truth-telling & District Bias \\
\hline LargeScale & $0.034 * *$ & -0.024 & -0.032 & $0.084 * * *$ & $-0.100 * * *$ & -0.047 \\
\hline & $(0.017)$ & $(0.046)$ & $(0.029)$ & $(0.020)$ & $(0.031)$ & $(0.032)$ \\
\hline DA & $0.115^{* * *}$ & & $-0.071 * *$ & $0.104 * * *$ & & -0.009 \\
\hline & $(0.020)$ & & $(0.035)$ & $(0.022)$ & & $(0.032)$ \\
\hline $\mathrm{DA} \times$ LargeScale & -0.001 & & -0.021 & -0.010 & & -0.060 \\
\hline & $(0.031)$ & & $(0.041)$ & $(0.034)$ & & $(0.047)$ \\
\hline Observations & 8,320 & 4,381 & 3,397 & 3,200 & 1,726 & 1,209 \\
\hline
\end{tabular}

Notes: Standard errors in parentheses are clustered at the session level for specification (1), (2) and (3), at subject level for specification (4), (5) and (6). *** $\mathrm{p}<0.01, * * \mathrm{p}<0.05, * \mathrm{p}<0.1$

Table 16: Probability of always playing best response after a certain period: probit regressions

\begin{tabular}{rcccc}
\hline Treatment & \multicolumn{2}{c}{ All-human } & \multicolumn{2}{c}{ Empirical-robots } \\
& $(1)$ & $(2)$ & $(3)$ & $(4)$ \\
\hline LargeScale & $0.040^{*}$ & $0.040^{*}$ & $0.147^{* *}$ & $0.155^{* *}$ \\
& $(0.021)$ & $(0.022)$ & $(0.067)$ & $(0.067)$ \\
DA & $0.196^{* * *}$ & $0.197 * * *$ & $0.207 * * *$ & $0.214^{* * *}$ \\
& $(0.067)$ & $(0.067)$ & $(0.063)$ & $(0.063)$ \\
DA $\times$ LargeScale & 0.113 & 0.111 & 0.013 & 0.002 \\
& $(0.091)$ & $(0.092)$ & $(0.102)$ & $(0.101)$ \\
Period & & $0.022^{* * *}$ & & $0.035^{* * *}$ \\
& & $(0.001)$ & & $(0.002)$ \\
Observations & 8,320 & 8,320 & 3,200 & 3,200 \\
\hline
\end{tabular}

Notes: Standard errors in parentheses are clustered at the session level for specification (1) and (2), at subject level for specification (3) and (4). *** $\mathrm{p}<0.01, * * \mathrm{p}<0.05, * \mathrm{p}<0.1$ 


\section{References}

Abdulkadiroğlu and Sönmez, T. (2003). School choice: A mechanism design approach. American Economic Review, 93(3):729-747.

Abdulkadiroğlu, A., Pathak, P., Roth, A. E., and Sönmez, T. (2006). Changing the Boston school choice mechanism. Working paper w11965, National Bureau of Economic Research.

Abdulkadiroğlu, A., Pathak, P. A., and Roth, A. E. (2005a). The New York City high school match. American Economic Review, 95(2):364-367.

Abdulkadiroğlu, A., Pathak, P. A., and Roth, A. E. (2009). Strategy-proofness versus efficiency in matching with indifferences: Redesigning the NYC high school match. American Economic Review, 99(5):1954-1978.

Abdulkadiroğlu, A., Pathak, P. A., Roth, A. E., and Sönmez, T. (2005b). The Boston public school match. American Economic Review, 95(2):368-371.

Ashlagi, I., Braverman, M., and Hassidim, A. (2011). Matching with couples revisited. In Proceedings of the 12th ACM conference on Electronic commerce, pages 335-336. ACM.

Ashlagi, I. and Roth, A. (2011). Individual rationality and participation in large scale, multihospital kidney exchange. In Proceedings of the 12th ACM conference on Electronic commerce, pages 321-322. ACM.

Azevedo, E. M. and Budish, E. (2013). Strategyproofness in the large. http://assets . wharton. upenn. edu/ eazevedo/papers/Azevedo-Budish-SPL.pdf.

Azevedo, E. M. and Hatfield, J. W. (2013). Complementarity and multidimensional heterogeneity in matching markets. http: / / assets.wharton.upenn.edu/ eazevedo/papers / Azevedo\%20Hatfield\%20-\%20Existence 200 f\%20Stable 20 Matchings\% 20with\%20 Complements.pdf.

Azevedo, E. M. and Leshno, J. D. (2011). Can we make school choice more efficient? Unpublished.

Azevedo, E. M. and Leshno, J. D. (2014). A supply and demand framework for two-sided matching markets. http://assets.wharton.upenn.edu/ eazevedo/papers / Azevedo-Leshno-Supply-and-Demand-Matching •pdf.

Bogomolnaia, A. and Moulin, H. (2001). A new solution to the random assignment problem. Journal of Economic Theory, 100(2):295-328. 
Calsamiglia, C., Haeringer, G., and Klijn, F. (2010). Constrained school choice: An experimental study. American Economic Review, 100(4):1860-74.

Che, Y.-K., Kim, J., and Kojima, F. (2013). Efficient assignment with interdependent values. http://www. columbia.edu/ yc2271/files/papers/CKK.pdf.

Chen, Y. and Kesten, O. (2014). Chinese college admissions and school choice reforms: Theory and experiments. http://yanchen.people.si.umich.edu/papers/Chen_ Kesten_2014_01.pdf.

Chen, Y. and Sönmez, T. (2002). Improving efficiency of on-campus housing: An experimental study. American Economic Review, 92(5):1669-1686.

Chen, Y. and Sönmez, T. (2004). An experimental study of house allocation mechanisms. Economic Letters, 83(1):137-140.

Chen, Y. and Sönmez, T. (2006). School choice: An experimental study. Journal of Economic Theory, 127:202-231.

Chen, Y. and Takeuchi, K. (2010). Multi-object auctions with package bidding: An experimental comparison of Vickrey and iBEA. Games and Economic Behavior, 68(2):557 - 579.

Cripps, M. W. and Swinkels, J. M. (2006). Efficiency of large double auctions. Econometrica, 74(1):47-92.

Davis, A. M., Katok, E., and Kwasnica, A. M. (2010). Do auctioneers pick optimal reserve prices? Management Science, pages 1-16.

Dubins, L. E. and Freedman, D. A. (1981). Machiavelli and the Gale-Shapley algorithm. American Mathematical Monthly, 88:485-494.

Ergin, H. and Sönmez, T. (2006). Games of school choice under the Boston mechanism. Journal of Public Economics, 90(1-2):215-237.

Featherstone, C. and Niederle, M. (2014). Improving on strategy-proof school choice mechanisms: An experimental investigation. http://assets.wharton.upenn.edu/ claytonf/ IncInfo14-02-01-2014.pdf.

Ferraro, P. J. and Vossler, C. A. (2010). The source and significance of confusion in public goods experiments. The B.E. Journal of Economic Analysis \& Policy, 10(1):Article 53. 
Fischbacher, U. (2007). z-tree: Zurich toolbox for ready-made economic experiments. Experimental Economics, 10:171-178.

Fudenberg, D., Mobius, M., and Szeidl, A. (2007). Existence of equilibrium in large double auctions. Journal of Economic theory, 133(1):550-567.

Gale, D. and Shapley, L. S. (1962). College admissions and the stability of marriage. American Mathematical Monthly, 69:9-15.

Gresik, T. A. and Satterthwaite, M. A. (1989). The rate at which a simple market converges to efficiency as the number of traders increases: An asymptotic result for optimal trading mechanisms. Journal of Economic Theory, 48(1):304-332.

Hatfield, J. W., Kojima, F., and Narita, Y. (2012). Promoting school competition through school choice: A market design approach. http://papers.ssrn.com/sol3/papers.cfm? abstract_id=1984876.

He, Y. (2014). Gaming the Boston school choice mechanism in Beijing. https: //26b054f3-a-62cb3ala-s-sites.googlegroups.com/site/yinghuahe/ research/papers/BM.pdf.

Hoxby, C. M. (2003). School choice and school productivity: Could school choice be a tide that lifts all boats? In Hoxby, C. M., editor, The Economics of School Choice. University of Chicago Press, Chicago.

Immorlica, N. and Mahdian, M. (2005). Marriage, honesty, and stability. In Proceedings of the Sixteenth Annual ACM-SIAM Symposium on Discrete Algorithms, SODA '05, pages 53-62, Philadelphia, PA, USA. Society for Industrial and Applied Mathematics.

Kagel, J. H. and Levin, D. (2001). Behavior in multi-unit demand auctions: Experiments with uniform price and dynamic Vickrey auctions. Econometrica, 69(2):413-454.

Kagel, J. H. and Roth, A. E. (2000). The dynamics of reorganization in matching markets: A laboratory experiment motivated by a natural experiment. Quarterly Journal of Economics, 115(1):201-235.

Klijn, F., Pais, J., and Vorsatz, M. (2013). Preference intensities and risk aversion in school choice: A laboratory experiment. Experimental Economics, 16(1):1-22.

Kojima, F. and Manea, M. (2010). Incentives in the probabilistic serial mechanism. Journal of Economic Theory, 145(1):106-123. 
Kojima, F. and Pathak, P. A. (2009). Incentives and stability in large two-sided matching markets. American Economic Review, 99:608-627.

Kojima, F., Pathak, P. A., and Roth, A. E. (2013). Matching with couples: Stability and incentives in large markets. The Quarterly Journal of Economics, 1(48):48.

Niederle, M. and Roth, A. E. (2003). Unraveling reduces mobility in a labor market: Gastroenterology with and without a centralized match. Journal of Political Economy, 111(6):1342-1352.

Pais, J. and Pintér, Á. (2008). School choice and information: An experimental study on matching mechanisms. Games and Economic Behavior, 64(1):303 - 328.

Pathak, P. A. and Sönmez, T. (2008). Leveling the playing field: sincere and sophisticated players in the boston mechanism. American Economic Review, 98:1636-1652(17).

Pathak, P. A. and Sönmez, T. (2013). School admissions reform in chicago and england: Comparing mechanisms by their vulnerability to manipulation. American Economic Review, 103(1):80106.

Pesendorfer, W. and Swinkels, J. M. (2000). Efficiency and information aggregation in auctions. American Economic Review, pages 499-525.

Roberts, D. J. and Postlewaite, A. (1976). The incentives for price-taking behavior in large exchange economies. Econometrica, 44(1):115-27.

Roth, A. E. (1982). The economics of matching: Stability and incentives. Mathematics of Operations Research, 7:617-628.

Roth, A. E. (2002). The economist as engineer: Game theory, experimentation, and computation as tools for design economics. Econometrica, 70:1341-1378.

Roth, A. E. and Peranson, E. (1999). The redesign of the matching market for american physicians: Some engineering aspects of economic design. American Economic Review, 89(4):748-780.

Roth, A. E., Sönmez, T., and Ünver, M. U. (2007). Efficient kidney exchange: Coincidence of wants in markets with compatibility-based preferences. The American economic review, pages $828-851$.

Roth, A. E., Sönmez, T., and Utku Ünver, M. (2005). Pairwise kidney exchange. Journal of Economic Theory, 125(2):151-188. 
Roth, A. E. and Sotomayor, M. (1990). Two-Sided Matching: A Study in Game-Theoretic Modeling and Analysis. Cambridge University Press, Cambridge.

Rustichini, A., Satterthwaite, M. A., and Williams, S. R. (1994). Convergence to efficiency in a simple market with incomplete information. Econometrica, 62(5):1041-63.

Swinkels, J. M. (2001). Efficiency of large private value auctions. Econometrica, 69(1):37-68. 